DEMOGRAPHIC RESEARCH

VOLUME 43, ARTICLE 17, PAGES 461-500 PUBLISHED 13 AUGUST 2020

https://www.demographic-research.org/Volumes/Vol43/17/

DOI: 10.4054/DemRes.2020.43.17

Research Article

The sibsize revolution in an international context: Declining social disparities in the number of siblings in 26 countries

Patrick Präg

Seongsoo Choi

\title{
Christiaan Monden
}

(C) 2020 Patrick Präg, Seongsoo Choi \& Christiaan Monden.

This open-access work is published under the terms of the Creative Commons Attribution 3.0 Germany (CC BY 3.0 DE), which permits use, reproduction, and distribution in any medium, provided the original author(s) and source are given credit.

See https://creativecommons.org/licenses/by/3.0/de/legalcode. 


\section{Contents}

1 Introduction $\quad 462$

2 Sibship size in the intergenerational transmission of inequality 464

3 Data and methods 465

3.1 Assessment of trends over time 472

$\begin{array}{lll}3.2 & \text { Replicability } & 473\end{array}$

$4 \quad$ Results 473

4.1 Average sibship size, prevalence of only-children, and prevalence 473

4.2 Disparities in sibship size $\quad 474$

$4.3 \quad$ Disparities in growing up as an only child 478

4.4 Disparities in growing up in large families 479

4.5 Disparities in sibship size, distinguishing between paternal and 483

$5 \quad$ Summary and discussion $\quad 486$

6 Acknowledgments 489

$\begin{array}{ll}\text { References } & 490\end{array}$ 


\title{
The sibsize revolution in an international context: Declining social disparities in the number of siblings in 26 countries
}

\author{
Patrick Präg ${ }^{1}$ \\ Seongsoo Choi $^{2}$ \\ Christiaan Monden ${ }^{3}$
}

\begin{abstract}
BACKGROUND

One's number of siblings is an important determinant of many life outcomes, such as educational attainment. In the last century the United States has experienced a 'sibsize revolution', in which sibship sizes declined, and which led to a convergence in family circumstances for children. Did this happen in other countries as well?
\end{abstract}

\section{OBJECTIVE}

This study examines the development of sibship size and social disparities in sibship size in low-fertility countries across the $20^{\text {th }}$ century.

\section{METHODS}

We analyze sibship size data collected from 111 nationally representative surveys conducted in 26 low-fertility countries across the $20^{\text {th }}$ century.

\section{RESULTS}

Average sibship sizes have declined in virtually all countries. Average sibship sizes are socially stratified, with smaller sibship sizes among higher-educated parents. This social disparity in sibship size has declined over time, indicating convergence in most countries. This convergence applies to large families, but not to only-child families.

\section{CONTRIBUTION}

Siblings are an understudied phenomenon in family demography, despite their growing importance in a time of increasingly complex family structures. Given the significance of sibship size for children's educational outcomes and overall life chances, decreasing social disparities in sibship size suggest greater equality in the intergenerational transmission of advantage.

${ }^{1}$ CREST, ENSAE Paris, France. Email: patrick.prag@sociology.ox.ac.uk.

${ }^{2}$ Department of Sociology, Yonsei University, Republic of Korea.

${ }^{3}$ Nuffield College and Department of Sociology, University of Oxford, UK 


\section{Introduction}

Sibship size, defined as the number of children in a child's sibling group, is a predictor of many important early- and later-life outcomes, ranging from greater survival chances as infants to greater educational attainment for those with fewer siblings (Björklund and Salvanes 2011; Blau and Duncan 1967; Steelman et al. 2002). Sibship size is crucial for childrens' access to familial resources and living conditions. Siblings play an important role in the development of children. McHale, Updegraff, and Whiteman (2012) report that in $2010,82 \%$ of US children under the age of 18 years lived with at least one sibling - a percentage that is higher than those living with a father figure. In times of increasingly complex family structures (Kalmijn et al. 2019), the presence of siblings, including adopted siblings and stepsiblings, is a remarkable constant in children's family lives. Children spend the majority of their discretionary time in activities with their siblings (Dunifon, Fomby, and Musick 2017). Growing up without siblings results in fewer social skills during childhood (Downey, Condron, and Yucel 2015). Siblings are also important beyond childhood, as family relationships with siblings are typically the longest lasting in an individual's life (Dunifon, Fomby, and Musick 2017).

A change in sibship size therefore has important implications for the changing role of family in children's wellbeing and life chances. Fahey (2017) identifies declines in sibship sizes in the United States since the 1970s, particularly among children of black and lower-educated mothers. The decline in sibship size improves children's living conditions so much that it presumably offsets the negative consequences of the parallel trend of lone parenthood. Fahey suggests that the decline in sibship size amounts to a "sibsize revolution." But has this revolution also taken place outside of the United States? While social disparities in family size were a prominent area of research in the first half of the $20^{\text {th }}$ century (Van Bavel 2010; Notestein 1936), the research focus in the second half shifted to family structures, such as out-of-wedlock births, divorce, and lone parenthood (Fahey 2017). To assess whether the sibsize revolution diagnosed by Fahey (2017) also took place outside of the United States, we examine disparities in sibship sizes by parental education in 26 countries across the $20^{\text {th }}$ century.

Besides changes and socioeconomic disparities in average sibship size, we also examine the heterogeneity in sibship sizes, focusing on only-children (those without siblings) and children from large families (those with four siblings or more). Scholarly attention to these aspects of family size has been limited, as much of the existing research conceives sibship size effects as being linear in nature, largely overlooking possible nonmonotonic patterns. The prevalence of only-children varies considerably across countries (Choi and Monden 2017). Choi and Monden (2017) show that when the share of only-children in a country is high the only-children tend to come from advantaged socioeconomic backgrounds. When only-children are rare in a country this 
pattern is reversed: only-children disproportionally come from less advantaged family backgrounds. Is there a trend towards more only-children? And are social disparities in only-child prevalence converging? The changing nature of large families is also an under-researched issue. Research on parity progression in middle- and high-income countries focuses primarily on lower-order parities (up to three; e.g., Duvander et al. 2019; Köppen and Trappe 2019; Nisén et al. 2018) and neglects larger families. However, how socioeconomic disparities that result from living in large families have changed has rarely been addressed in prior demographic and sociological research, particularly in a comparative perspective.

In this study we make use of survey data on sibship size collected from 111 surveys conducted in 26 low-fertility countries over the course of the $20^{\text {th }}$ century. We are primarily interested in what happens to sibship size and the social stratification of sibship size over time, focusing on trends rather than point estimates. We focus on countries that had low fertility - broadly defined as a total fertility rate below 2.1 during the 1990s when the youngest cohorts in our samples were of school-age. Our sample includes most industrialized countries in Europe, North America, Australia, and East Asia, as well as many post-communist countries. We excluded countries in other regions (e.g., Latin America, South and Southeast Asia, Africa) that were either less developed or developing during the 1990s. We include some countries that transitioned to low fertility status relatively recently, such as some East Asian and post-communist countries.

In a first step we describe the development of average sibship sizes and the prevalence of only-children families and large families over the $20^{\text {th }}$ century. In a second step we compare social disparities in average sibship sizes, the prevalence of only-children, and the prevalence of large families. We assess social disparities by comparing outcomes by parental education. In a third step we examine disparities in sibship size distinguishing between paternal and maternal education. In an important study on Nordic countries, Jalovaara et al. (2019) find gender differences in educational disparities in both cohort fertility and childlessness. Our data allows us to assess differences in average sibship sizes by parental gender for a wider range of countries.

Our study makes three contributions to the existing literature. First, our descriptive account of sibship size over time and across countries is key for understanding the living conditions of children. McHale, Updegraff, and Whiteman (2012) criticize the lack of research on siblings in family demography and called for greater efforts to incorporate sibling relationships in research. Second, rather than just focusing on the average sibship size, we incorporate a wider range of family size indicators - onlychildren and large families - to better grasp the development of family size over time. Finally, our study adds to the growing body of interdisciplinary stratification research 
that seeks to incorporate intergenerational reproduction and demographic processes (Breen, Ermisch, and Helske 2019; Grätz et al. 2019; Song and Campbell 2017).

\section{Sibship size in the intergenerational transmission of inequality}

Sibship size has long been seen as an important factor predicting many child outcomes, such as survival, education, earnings, and occupational attainment (Steelman et al. 2002). Blake (1981) suggests that growing up in a large family makes it more difficult to access material and nonmaterial family resources, such as living space and books, trips to the museum and family vacations, or parental attention and intervention. Given that these resources are important inputs for children's developmental outcomes, on average children that share family resources with more siblings are expected to have worse educational outcomes. This 'resource dilution' model of child development has found much support in empirical research (see e.g., Gibbs, Workman, and Downey 2016; Workman 2016 for references).

Much of the existing research on sibship-size effects is correlational rather than causal in nature (Choi et al. 2020). Parental characteristics, for instance, could affect both family size and child wellbeing. As parental characteristics such as preferences, self-control, or planning abilities are usually unobserved, the association between family size and child outcomes is likely biased upward due to this confounding. Existing causal evidence on sibship-size effects points in that direction. Some studies, such as Black, Devereux, and Salvanes (2005) and Angrist, Lavy, and Schlosser (2010), make use of the sex composition of children and twinning as instrumental variables to account for unobserved confounding, and do not find consistent support for a causal sibship-size effect on education. Similarly designed studies, such as Conley and Glauber (2006), Ferrari and Dalla-Zuanna (2010), and Blaabæk, Jæger, and Molitoris (2020), find in a number of settings that only part of the negative association between sibship size and education is causal.

Family size is not only related to educational attainment but also to other important living conditions and outcomes in child development, both material and nonmaterial, such as housing conditions on the one hand, and sibling rivalry, reaction to stress, and learning leadership and cooperation on the other (Ernst and Angst 1983; McHale, Updegraff, and Whiteman 2012; Zajonc and Markus 1975). Sibling interactions are believed to be useful for developing social skills and relations with peers (Whiteman, McHale, and Soli 2011), and having fewer siblings - or no siblings at all - can have grave behavioral consequences (Downey, Condron, and Yucel 2015). For instance, Cameron et al. (2013) describe Chinese only-children as less trusting, less trustworthy, 
more risk-averse, less competitive, more pessimistic, and less conscientious than their counterparts who have siblings.

But sibship size is not only an antecedent of social inequality; sibship size itself is also a socially stratified phenomenon. Empirical research has long been occupied with studying fertility differentials between the rich and the poor (e.g., Dribe, Oris, and Pozzi 2014; Sobotka 2017). In contemporary societies, education often stands in the way of childbearing (and vice versa) (Balbo, Billari, and Mills 2013), often leaving the families of higher-educated parents smaller than those of their lower-educated counterparts, essentially creating a "two-tier family structure" for children (Putnam 2015). Children from a lower-educated family background often have to compete with more siblings for fewer resources than the children of higher-educated parents, potentially creating a downward spiral for those at the bottom of the educational distribution.

This disparity has not always existed in this form (Skirbekk 2008). For instance, Clark (2007) prominently argues that higher fertility among the British upper class was an important driver of the Industrial Revolution. Yet under conditions where out-ofwedlock births were rare and frowned upon, this comparatively high fertility among those of high social status partially stems from status differences in the timing of marriage and the proportion remaining unmarried (Hajnal 1965). Those of higher status married younger and thus had more time to have children, while those of lower status often remained unmarried and childless. Thus, the focus on fertility - family size seen from the mother's perspective - rather than sibship size - the perspective of the children - gives limited insight into sibship size and the actual living conditions of children growing up.

In sum, sibship size can be expected to be a major linchpin in the intergenerational transmission of inequality, as it is not only a predictor but also a consequence of lower education.

\section{Data and methods}

For our analyses we harmonized data from 111 national surveys collected in 26 countries. We have made this data publicly available as the International Sibsize and Educational Attainment Database (ISEAD, Monden et al. 2018). To create ISEAD we used the following criteria to select the surveys. First, we only utilized surveys from countries broadly defined as having low fertility at the end of the $20^{\text {th }}$ century. Our data includes countries in Europe (Western and Eastern, including the former Soviet Union), North America and Australia, and East Asia. Second, a survey had to be nationally representative of the adult population. We excluded studies surveying exclusively young children and adolescents because of the concern that the number of siblings 
might increase, as the respondents' parents might have more children in the future. Third, a survey had to contain all the information about a respondent necessary for our analysis, such as the number of siblings, parental educational attainment (father's or mother's, depending on which is higher, or father's if mother's education is unavailable), as well as basic demographic characteristics (e.g., age at the time of survey or year of birth, and sex). We excluded surveys containing incomplete information on sibship size. For example, we excluded several household-based surveys that only provided information on siblings living in the same household. Fourth, we only used surveys that covered at least 500 individuals.

Table 1 lists the surveys included in our analyses, stating sample size and respondents' age and birth cohort range. More comprehensive and general information, including files for data processing and macro-level data sets generated from the ISEAD, are available in Monden et al. (2018). We limit our sample to individuals who were at least 18 years old at the time of the survey. Because we take a cohort-based approach, we pooled all national surveys of each country and divided each pooled national sample into 9 ten-year-wide birth cohorts that cover the $20^{\text {th }}$ century: from those born between 1901 and 1910 to those born between 1981 and 1990. For robust estimation, we only used country-cohort samples with at least 500 observations. ${ }^{4}$ Taking into account these sample restrictions, the final data we used comprised 636,454 adult individuals from 179 country-cohort samples, as shown in Table 2.

Table 1: List of survey datasets in Monden et al. (2018) with information on sex composition, age/cohort, and missing observations ${ }^{5}$

\begin{tabular}{|c|c|c|c|c|c|c|c|c|c|}
\hline Country & Data set & Year & $\begin{array}{c}\% \\
\text { female }\end{array}$ & $\begin{array}{c}\text { Age } \\
\text { range }\end{array}$ & $\begin{array}{l}\text { Birth } \\
\text { cohort } \\
\text { range }\end{array}$ & $N$ full & $\begin{array}{l}N \text { cohort } \\
\text { restriction }\end{array}$ & $\begin{array}{l}N \text { parental } \\
\text { education }\end{array}$ & $\begin{array}{l}N \text { materna } \\
\text { education }\end{array}$ \\
\hline Australia & $\begin{array}{l}\text { International Social Survey } \\
\text { Program (ISSP Research Group } \\
\text { 1988) }\end{array}$ & 1986 & 52.1 & 18-84 & $\begin{array}{c}1902- \\
1968\end{array}$ & 2,500 & 2,156 & - & - \\
\hline Australia & $\begin{array}{l}\text { Generations and Gender } \\
\text { Program (Vikat et al. 2007) }\end{array}$ & 2005 & 55.4 & 18-98 & $\begin{array}{c}1906- \\
1987\end{array}$ & 7,125 & 6,871 & 6,129 & 5,705 \\
\hline Belgium & $\begin{array}{l}\text { Generations and Gender } \\
\text { Program (Vikat et al. 2007) }\end{array}$ & 2008 & 52.0 & 18-82 & $\begin{array}{l}1928- \\
1990\end{array}$ & 7,163 & 7,019 & 6,525 & 6,342 \\
\hline
\end{tabular}

\footnotetext{
${ }^{4}$ With one exception: the Spanish 1931-1940 birth cohort only has 495 observations.

5 " $N$ full" states the sample size of the entire survey data set, " $N$ cohort restriction" is the sample size after removing cases which were from birth cohorts before 1900 and cases with missing information about sibship size or birth year, " $N$ parental education" additionally removes cases where no information about the education of either parent was available. " $N$ maternal education" removes all cases where no information about maternal education was available. (The sample sizes for cases with missing paternal education is not shown, as it is almost identical to " $N$ parental education.")
} 
Table 1: (Continued)

\begin{tabular}{|c|c|c|c|c|c|c|c|c|c|}
\hline Country & Data set & Year & $\begin{array}{c}\% \\
\text { female }\end{array}$ & $\begin{array}{l}\text { Age } \\
\text { range }\end{array}$ & $\begin{array}{l}\text { Birth } \\
\text { cohort } \\
\text { range }\end{array}$ & $N$ full & $\begin{array}{l}N \text { cohort } \\
\text { restriction }\end{array}$ & $\begin{array}{l}N \text { parental } \\
\text { education }\end{array}$ & $\begin{array}{c}N \text { maternal } \\
\text { education }\end{array}$ \\
\hline Bulgaria & $\begin{array}{l}\text { Social Stratification in Eastern } \\
\text { Europe (Szelényi and Treiman } \\
\text { 2017) }\end{array}$ & 1993 & 51.7 & 19-78 & $\begin{array}{l}1915- \\
1974\end{array}$ & 4,919 & 4,652 & 4,577 & 4,488 \\
\hline Bulgaria & $\begin{array}{l}\text { Generations and Gender } \\
\text { Program (Vikat et al. 2007) }\end{array}$ & 2004 & 54.5 & 18-85 & $\begin{array}{c}1919- \\
1986\end{array}$ & 12,858 & 12,206 & 11,726 & 11,560 \\
\hline Canada & $\begin{array}{l}\text { Class Structure and Class } \\
\text { Consciousness Survey (Wright } \\
\text { 1990) }\end{array}$ & 1982 & 48.2 & 18-80 & $\begin{array}{c}1902- \\
1964\end{array}$ & 2,577 & 2,554 & 1,162 & 1,120 \\
\hline Canada & $\begin{array}{l}\text { Canadian General Social } \\
\text { Survey (Statistics Canada 1995) }\end{array}$ & 1995 & 55.0 & 18-80 & $\begin{array}{c}1915- \\
1977\end{array}$ & 10,749 & 10,303 & 9,075 & 8,545 \\
\hline Canada & $\begin{array}{l}\text { Canadian General Social } \\
\text { Survey (Statistics Canada 2000) }\end{array}$ & 2000 & 56.1 & $18-80$ & $\begin{array}{c}1920- \\
1982\end{array}$ & 24,310 & 23,454 & 20,167 & 18,949 \\
\hline China & $\begin{array}{l}\text { China Housing Survey (Logan } \\
\text { and Bian 2000) }\end{array}$ & 1993 & 39.7 & 18-67 & $\begin{array}{c}1931- \\
1980\end{array}$ & 2,096 & 1,857 & 1,108 & 993 \\
\hline China & $\begin{array}{l}\text { East Asian Social Survey (Kim } \\
\text { et al. 2014) }\end{array}$ & 2006 & 54.7 & 18-69 & $\begin{array}{c}1937- \\
1988\end{array}$ & 3,208 & 3,206 & 3,202 & 3,200 \\
\hline China & $\begin{array}{l}\text { China Family Panel Studies (Xie } \\
\text { et al. 2016) }\end{array}$ & 2010 & 51.7 & $20-79$ & $\begin{array}{c}1931- \\
1990\end{array}$ & 31,731 & 30,965 & 27,674 & 26,950 \\
\hline Czechia & $\begin{array}{l}\text { Social Stratification in Eastern } \\
\text { Europe (Szelényi and Treiman } \\
\text { 2017) }\end{array}$ & 1993 & 55.0 & $20-70$ & $\begin{array}{c}1923- \\
1973\end{array}$ & 5,621 & 5,579 & 5,500 & 5,409 \\
\hline Czechia & $\begin{array}{l}\text { Generations and Gender } \\
\text { Program (Vikat et al. 2007) }\end{array}$ & 2005 & 52.1 & 18-79 & $\begin{array}{c}1926- \\
1987\end{array}$ & 10,006 & 9,939 & 9,305 & 9,211 \\
\hline $\begin{array}{l}\text { East } \\
\text { Germany }\end{array}$ & $\begin{array}{l}\text { German Socio-Economic Panel } \\
\text { (Schupp et al. 2017) }\end{array}$ & $\begin{array}{c}1984- \\
2015\end{array}$ & 52.3 & 18-95 & $\begin{array}{c}1902- \\
1990\end{array}$ & 14,730 & 9,260 & 8,652 & 8,322 \\
\hline $\begin{array}{l}\text { East } \\
\text { Germany }\end{array}$ & $\begin{array}{l}\text { Generations and Gender } \\
\text { Program (Vikat et al. 2007) }\end{array}$ & 2005 & 57.0 & $18-85$ & $\begin{array}{c}1920- \\
1987\end{array}$ & 1,766 & 1,736 & 1,661 & 1,659 \\
\hline Estonia & $\begin{array}{l}\text { Generations and Gender } \\
\text { Program (Vikat et al. 2007) }\end{array}$ & 2005 & 64.1 & $20-81$ & $\begin{array}{c}1924- \\
1983\end{array}$ & 7,855 & 7,290 & 7,278 & 7,275 \\
\hline France & $\begin{array}{l}\text { Training and Qualifying Survey } \\
\text { Professional (INSEE 1985) }\end{array}$ & 1985 & 42.7 & 18-84 & $\begin{array}{c}1901- \\
1967\end{array}$ & 39,233 & 38,446 & 38,005 & 37,528 \\
\hline France & $\begin{array}{l}\text { Training and Qualifying Survey } \\
\text { Professional (INSEE 1993) }\end{array}$ & 1993 & 50.7 & $20-64$ & $\begin{array}{c}1929- \\
1973\end{array}$ & 18,332 & 17,986 & 17,814 & 17,634 \\
\hline France & $\begin{array}{l}\text { Training and Qualifying Survey } \\
\text { Professional (INSEE 2003) }\end{array}$ & 2003 & 52.8 & $18-65$ & $\begin{array}{c}1938- \\
1985\end{array}$ & 39,285 & 39,285 & 39,026 & 38,830 \\
\hline France & $\begin{array}{l}\text { Generations and Gender } \\
\text { Program (Vikat et al. 2007) }\end{array}$ & 2005 & 56.6 & 18-79 & $\begin{array}{c}1926- \\
1987\end{array}$ & 10,079 & 10,039 & 8,983 & 8,631 \\
\hline Georgia & $\begin{array}{l}\text { Generations and Gender } \\
\text { Program (Vikat et al. 2007) }\end{array}$ & 2006 & 55.9 & $18-80$ & $\begin{array}{c}1926- \\
1988\end{array}$ & 10,000 & 9,547 & 9,101 & 8,959 \\
\hline Hungary & $\begin{array}{l}\text { International Social Survey } \\
\text { Program (ISSP Research Group } \\
\text { 1988) }\end{array}$ & 1986 & 55.9 & $18-85$ & $\begin{array}{c}1901- \\
1968\end{array}$ & 1,747 & 1,626 & - & - \\
\hline Hungary & $\begin{array}{l}\text { Social Stratification in Eastern } \\
\text { Europe (Szelényi and Treiman } \\
\text { 2017) }\end{array}$ & 1993 & 53.4 & 18-92 & $\begin{array}{l}1901- \\
1975\end{array}$ & 4,977 & 4,418 & - & - \\
\hline Hungary & $\begin{array}{l}\text { Generations and Gender } \\
\text { Program (Vikat et al. 2007) }\end{array}$ & 2004 & 55.5 & $20-79$ & $\begin{array}{c}1926- \\
1983\end{array}$ & 13,540 & 13,505 & 13,353 & 13,282 \\
\hline
\end{tabular}


Präg, Choi \& Monden: The sibsize revolution in international context

Table 1: (Continued)

\begin{tabular}{|c|c|c|c|c|c|c|c|c|c|}
\hline Country & Data set & Year & $\begin{array}{c}\% \\
\text { female }\end{array}$ & $\begin{array}{l}\text { Age } \\
\text { range }\end{array}$ & $\begin{array}{l}\text { Birth } \\
\text { cohort } \\
\text { range }\end{array}$ & $\mathrm{N}$ full & $\begin{array}{l}N \text { cohort } \\
\text { restriction }\end{array}$ & $\begin{array}{c}N \\
\text { parental } \\
\text { education }\end{array}$ & $\begin{array}{l}N \text { maternal } \\
\text { education }\end{array}$ \\
\hline Italy & $\begin{array}{l}\text { Generations and Gender Program } \\
\text { (Vikat et al. 2007) }\end{array}$ & 2003 & 53.4 & $18-64$ & $\begin{array}{c}1939- \\
1985\end{array}$ & 9,570 & 9,570 & 9,366 & 9,338 \\
\hline Japan & $\begin{array}{l}\text { Japanese Social Stratification and } \\
\text { Mobility (SSM Survey Management } \\
\text { Committee 2011a) }\end{array}$ & 1995 & 53.5 & $20-70$ & $\begin{array}{l}1925- \\
1975\end{array}$ & 5,357 & 5,351 & 4,985 & 4,545 \\
\hline Japan & $\begin{array}{l}\text { Japanese General Social Survey } \\
\text { (Tanioka, Iwai, et al. 2007a) }\end{array}$ & 2000 & 54.4 & $20-89$ & $\begin{array}{c}1911- \\
1980\end{array}$ & 2,893 & 2,792 & 2,374 & 2,317 \\
\hline Japan & $\begin{array}{l}\text { National Survey on Family and } \\
\text { Economic Conditions (Tsuya et al. } \\
\text { 2009) }\end{array}$ & 2000 & 53.1 & $20-50$ & $\begin{array}{l}1950- \\
1980\end{array}$ & 4,482 & 3,357 & - & - \\
\hline Japan & $\begin{array}{l}\text { Japanese General Social Survey } \\
\text { (Tanioka, Iwai, et al. 2007b) }\end{array}$ & 2001 & 54.0 & $20-89$ & $\begin{array}{c}1912- \\
1981\end{array}$ & 2,790 & 2,653 & 2,229 & 2,180 \\
\hline Japan & $\begin{array}{l}\text { Japanese General Social Survey } \\
\text { (Tanioka, Iwai, et al. 2007c) }\end{array}$ & 2002 & 53.7 & $20-89$ & $\begin{array}{c}1913- \\
1982\end{array}$ & 2,953 & 2,882 & 2,449 & 2,401 \\
\hline Japan & $\begin{array}{l}\text { Japanese General Social Survey } \\
\text { (Tanioka, Nitta, et al. 2007) }\end{array}$ & 2005 & 54.5 & $20-89$ & $\begin{array}{c}1916- \\
1985\end{array}$ & 2,023 & 1,997 & 1,553 & 1,529 \\
\hline Japan & $\begin{array}{l}\text { Japanese Social Stratification and } \\
\text { Mobility (SSM Survey Management } \\
\text { Committee 2011b) }\end{array}$ & 2005 & 53.7 & $20-70$ & $\begin{array}{l}1935- \\
1985\end{array}$ & 5,742 & 5,726 & 4,781 & 4,572 \\
\hline Japan & $\begin{array}{l}\text { East Asian Social Survey (Kim et al. } \\
\text { 2014) }\end{array}$ & 2006 & 54.7 & $20-89$ & $\begin{array}{c}1917- \\
1986\end{array}$ & 2,130 & 2,097 & 1,665 & 1,613 \\
\hline Japan & $\begin{array}{l}\text { Japanese General Social Survey } \\
\text { (Tanioka et al. 2010) }\end{array}$ & 2006 & 53.3 & 20-89 & $\begin{array}{c}1917- \\
1986\end{array}$ & 4,254 & 4,192 & 3,349 & 3,246 \\
\hline Japan & $\begin{array}{l}\text { Japanese General Social Survey } \\
\text { (Tanioka et al. 2015) }\end{array}$ & 2008 & 52.9 & $20-89$ &. & 4,220 & 4,204 & 3,491 & 3,404 \\
\hline Japan & $\begin{array}{l}\text { National Family Research of Japan } \\
\text { (Inaba 2015) }\end{array}$ & 2008 & 52.9 & $28-73$ & $\begin{array}{c}1935- \\
1980\end{array}$ & 5,203 & 5,115 & 4,327 & 4,202 \\
\hline Japan & $\begin{array}{l}\text { National Survey on Family and } \\
\text { Economic Conditions (Choe et al. } \\
\text { 2013) }\end{array}$ & 2009 & 53.0 & $20-49$ & $\begin{array}{c}1960- \\
1989\end{array}$ & 3,112 & 2,632 & 2,463 & 2,406 \\
\hline Japan & $\begin{array}{l}\text { Japanese General Social Survey } \\
\text { (Tanioka, Maeda, and Iwai 2015) }\end{array}$ & 2010 & 54.0 & $20-89$ & $\begin{array}{c}1921- \\
1990\end{array}$ & 5,003 & 4,975 & 4,103 & 4,018 \\
\hline Japan & $\begin{array}{l}\text { Japanese General Social Survey } \\
\text { (Tanioka, Iwai, and Maeda 2016) }\end{array}$ & 2012 & 54.0 & $22-89$ & $\begin{array}{c}1923- \\
1990\end{array}$ & 4,667 & 4,566 & 3,700 & 3,584 \\
\hline Lithuania & $\begin{array}{l}\text { Generations and Gender Program } \\
\text { (Vikat et al. 2007) }\end{array}$ & 2006 & 50.2 & $18-79$ & $\begin{array}{c}1926- \\
1988\end{array}$ & 10,036 & 9,543 & 8,544 & 8,329 \\
\hline $\begin{array}{l}\text { Nether- } \\
\text { lands }\end{array}$ & $\begin{array}{l}\text { Family Survey Dutch Population } \\
\text { (Ganzeboom and Ultee 1993) }\end{array}$ & 1992 & 51.1 & $18-78$ & $\begin{array}{c}1914- \\
1974\end{array}$ & 1,801 & 1,790 & 1,786 & 1,764 \\
\hline $\begin{array}{l}\text { Nether- } \\
\text { lands }\end{array}$ & $\begin{array}{l}\text { Family Survey Dutch Population (De } \\
\text { Graaf et al. 1998) }\end{array}$ & 1998 & 50.7 & $18-83$ & $\begin{array}{c}1915- \\
1980\end{array}$ & 2,029 & 2,027 & 2,004 & 1,992 \\
\hline $\begin{array}{l}\text { Nether- } \\
\text { lands }\end{array}$ & $\begin{array}{l}\text { Family Survey Dutch Population (De } \\
\text { Graaf et al. 2000) }\end{array}$ & 2000 & 50.1 & $18-84$ & $\begin{array}{c}1916- \\
1982\end{array}$ & 1,561 & 1,539 & 1,530 & 1,511 \\
\hline $\begin{array}{l}\text { Nether- } \\
\text { lands }\end{array}$ & $\begin{array}{l}\text { Family Survey Dutch Population (De } \\
\text { Graaf et al. 2004) }\end{array}$ & 2003 & 58.1 & $18-80$ & $\begin{array}{c}1923- \\
1985\end{array}$ & 8,161 & 7,875 & 7,137 & 6,979 \\
\hline
\end{tabular}


Table 1: (Continued)

\begin{tabular}{|c|c|c|c|c|c|c|c|c|c|}
\hline Country & Data set & Year & $\begin{array}{c}\% \\
\text { female }\end{array}$ & $\begin{array}{l}\text { Age } \\
\text { range }\end{array}$ & $\begin{array}{l}\text { Birth } \\
\text { cohort } \\
\text { range }\end{array}$ & $\mathrm{N}$ full & $\begin{array}{l}N \text { cohort } \\
\text { restriction }\end{array}$ & $\begin{array}{c} \\
\text { parental } \\
\text { education }\end{array}$ & $\begin{array}{l}N \text { maternal } \\
\text { education }\end{array}$ \\
\hline $\begin{array}{l}\text { Nether- } \\
\text { lands }\end{array}$ & $\begin{array}{l}\text { Generations and Gender Program } \\
\text { (Vikat et al. 2007) }\end{array}$ & 2003 & 51.1 & $18-79$ & $\begin{array}{c}1924- \\
1985\end{array}$ & 2,174 & 2,123 & 2,083 & 2,042 \\
\hline Norway & $\begin{array}{l}\text { Generations and Gender Program } \\
\text { (Vikat et al. 2007) }\end{array}$ & 2007 & 50.7 & 18-81 & $\begin{array}{c}1927- \\
1988\end{array}$ & 14,880 & 13,876 & 13,631 & 13,538 \\
\hline Poland & $\begin{array}{l}\text { Polish Panel Survey (Słomczynski et } \\
\text { al. 2008) }\end{array}$ & 1988 & 53.9 & $19-68$ & $\begin{array}{c}1920- \\
1969\end{array}$ & 5,817 & 1,888 & 1,855 & - \\
\hline Poland & $\begin{array}{l}\text { Social Stratification in Eastern Europe } \\
\text { (Szelényi and Treiman 2017) }\end{array}$ & 1993 & 52.3 & 19-77 & $\begin{array}{c}1916- \\
1974\end{array}$ & 3,520 & 3,439 & 3,383 & 3,317 \\
\hline Poland & $\begin{array}{l}\text { Polish General Social Survey } \\
\text { (Cichomski, Jerzynski, and Zielinski } \\
\text { 2007) }\end{array}$ & 1997 & 56.2 & 18-92 & $\begin{array}{c}1905- \\
1979\end{array}$ & 2,401 & 1,134 & 1,101 & 1,065 \\
\hline Poland & $\begin{array}{l}\text { Polish General Social Survey } \\
\text { (Cichomski, Jerzynski, and Zielinski } \\
\text { 2007) }\end{array}$ & 1999 & 56.9 & 18-94 & $\begin{array}{l}1905- \\
1981\end{array}$ & 2,282 & 2,200 & 2,158 & 2,115 \\
\hline Poland & $\begin{array}{l}\text { Polish General Social Survey } \\
\text { (Cichomski, Jerzynski, and Zielinski } \\
\text { 2007) }\end{array}$ & 2002 & 57.6 & 18-94 & $\begin{array}{c}1908- \\
1984\end{array}$ & 2,473 & 2,410 & 2,395 & 2,354 \\
\hline Poland & $\begin{array}{l}\text { Generations and Gender Program } \\
\text { (Vikat et al. 2007) }\end{array}$ & 2010 & 57.9 & 19-83 & $\begin{array}{c}1927- \\
1990\end{array}$ & 19,987 & 18,550 & 17,473 & 17,207 \\
\hline Romania & $\begin{array}{l}\text { Generations and Gender Program } \\
\text { (Vikat et al. 2007) }\end{array}$ & 2005 & 50.1 & $18-80$ & $\begin{array}{c}1925- \\
1987\end{array}$ & 11,986 & 11,986 & 11,759 & 11,588 \\
\hline Russia & $\begin{array}{l}\text { Russian General Social Survey } \\
\text { (Swafford et al. 2006) }\end{array}$ & 1992 & 55.3 & 18-89 & $\begin{array}{c}1903- \\
1974\end{array}$ & 2,149 & 1,948 & - & - \\
\hline Russia & $\begin{array}{l}\text { Social Stratification in Eastern Europe } \\
\text { (Szelényi and Treiman 2017) }\end{array}$ & 1993 & 59.9 & 18-89 & $\begin{array}{c}1904- \\
1975\end{array}$ & 5,002 & 4,364 & 4,301 & 4,246 \\
\hline Russia & $\begin{array}{l}\text { Generations and Gender Program } \\
\text { (Vikat et al. 2007) }\end{array}$ & 2004 & 62.5 & 18-81 & $\begin{array}{c}1923- \\
1986\end{array}$ & 11,261 & 10,989 & 9,522 & 8,988 \\
\hline $\begin{array}{l}\text { South } \\
\text { Korea }\end{array}$ & $\begin{array}{l}\text { Inequality and Equity Survey (Whang } \\
\text { et al. 2004) }\end{array}$ & 1990 & 20.2 & 18-83 & $\begin{array}{c}1907- \\
1972\end{array}$ & 1,974 & 1,846 & 1,843 & 1,841 \\
\hline $\begin{array}{l}\text { South } \\
\text { Korea }\end{array}$ & $\begin{array}{l}\text { Korean General Social Survey (Kim } \\
\text { 2014) }\end{array}$ & 2004 & 53.4 & 18-93 & $\begin{array}{c}1911- \\
1986\end{array}$ & 1,312 & 1,260 & 1,195 & 1,178 \\
\hline $\begin{array}{l}\text { South } \\
\text { Korea }\end{array}$ & $\begin{array}{l}\text { Korean General Social Survey (Kim et } \\
\text { al. 2014) }\end{array}$ & 2006 & 55.5 & 18-92 & $\begin{array}{c}1914- \\
1988\end{array}$ & 1,605 & 1,551 & 1,469 & 1,426 \\
\hline $\begin{array}{l}\text { South } \\
\text { Korea }\end{array}$ & $\begin{array}{l}\text { Education and Social Mobility Survey } \\
\text { (Park 2011) }\end{array}$ & 2009 & 49.4 & $25-65$ & $\begin{array}{c}1943- \\
1986\end{array}$ & 7,616 & 7,610 & 7,454 & 7,396 \\
\hline Spain & $\begin{array}{l}\text { Occupational Prestige and Social } \\
\text { Structure (CIS 2013) }\end{array}$ & 2013 & 51.0 & 23-99 & $\begin{array}{c}1914- \\
1990\end{array}$ & 5,962 & 5,271 & 5,195 & 5,160 \\
\hline Sweden & $\begin{array}{l}\text { Generations and Gender Program } \\
\text { (Vikat et al. 2007) }\end{array}$ & 2012 & 51.5 & $21-80$ & $\begin{array}{c}1933- \\
1990\end{array}$ & 9,688 & 8,964 & 8,319 & 7,930 \\
\hline Taiwan & $\begin{array}{l}\text { Taiwan Social Change Survey (Chiu } \\
\text { 1999) }\end{array}$ & 1997 & 52.6 & $20-64$ & $\begin{array}{c}1933- \\
1977\end{array}$ & 1,717 & 1,717 & 1,681 & 1,665 \\
\hline Taiwan & $\begin{array}{l}\text { Panel Study of Chinese Family } \\
\text { Dynamics (Chu 2002) }\end{array}$ & 1999 & 54.8 & $36-46$ & $\begin{array}{c}1953- \\
1963\end{array}$ & 999 & 995 & 973 & 961 \\
\hline Taiwan & $\begin{array}{l}\text { Taiwan Social Change Survey (Chang } \\
\text { 2002) }\end{array}$ & 2001 & 49.5 & 21-93 & $\begin{array}{c}1902- \\
1974\end{array}$ & 1,979 & 1,870 & 1,828 & 1,817 \\
\hline
\end{tabular}


Präg, Choi \& Monden: The sibsize revolution in international context

Table 1: (Continued)

\begin{tabular}{|c|c|c|c|c|c|c|c|c|c|}
\hline Country & Data set & Year & $\begin{array}{c}\% \\
\text { female }\end{array}$ & $\begin{array}{l}\text { Age } \\
\text { range }\end{array}$ & $\begin{array}{l}\text { Birth } \\
\text { cohort } \\
\text { range }\end{array}$ & $\mathrm{N}$ full & $\begin{array}{l}N \text { cohort } \\
\text { restriction }\end{array}$ & $\begin{array}{c}N \\
\text { parental } \\
\text { education }\end{array}$ & $\begin{array}{l}N \text { materna } \\
\text { education }\end{array}$ \\
\hline Taiwan & $\begin{array}{l}\text { Taiwan Social Change Survey (Chang } \\
\text { 2014) }\end{array}$ & 2004 & 48.6 & 19-98 & $\begin{array}{c}1906- \\
1985\end{array}$ & 1,781 & 1,755 & 1,705 & 1,693 \\
\hline Taiwan & $\begin{array}{l}\text { East Asian Social Survey (Kim et al. } \\
\text { 2014) }\end{array}$ & 2006 & 49.8 & 19-92 & $\begin{array}{c}1903- \\
1976\end{array}$ & 2,102 & 1,974 & 1,948 & 1,936 \\
\hline Taiwan & $\begin{array}{l}\text { Taiwan Social Change Survey (Chang } \\
\text { 2016) }\end{array}$ & 2012 & 49.7 & 22-101 & $\begin{array}{c}1911- \\
1990\end{array}$ & 2,134 & 2,001 & 1,928 & 1,880 \\
\hline Taiwan & $\begin{array}{l}\text { Taiwan Social Change Survey (Fu } \\
\text { 2015) }\end{array}$ & 2014 & 50.2 & $24-97$ & $\begin{array}{c}1917- \\
1990\end{array}$ & 1,875 & 1,695 & 1,643 & 1,615 \\
\hline Taiwan & $\begin{array}{l}\text { Taiwan Social Change Survey (Fu } \\
\text { 2017) }\end{array}$ & 2015 & 48.8 & 25-94 & $\begin{array}{c}1921- \\
1990\end{array}$ & 2,034 & 1,809 & 1,744 & 1,716 \\
\hline USA & $\begin{array}{l}\text { Growth of American Families } \\
\text { (Freedman, Campbell, and Whelpton } \\
\text { 2009) }\end{array}$ & 1955 & 100.0 & $18-54$ & $\begin{array}{c}1901- \\
1937\end{array}$ & 2,713 & 2,661 & - & - \\
\hline USA & $\begin{array}{l}\text { General Social Survey (Smith, Hout, } \\
\text { and Marsden 2016) }\end{array}$ & $\begin{array}{c}1972- \\
2014\end{array}$ & 55.9 & $18-89$ & $\begin{array}{c}1901- \\
1990\end{array}$ & 59,599 & 56,675 & 50,816 & 47,732 \\
\hline $\begin{array}{l}\text { United } \\
\text { Kingdom }\end{array}$ & $\begin{array}{l}\text { Oxford Social Mobility Inquiry (Oxford } \\
\text { Social Mobility Group et al. 1978) }\end{array}$ & 1972 & 0.0 & $20-64$ & $\begin{array}{c}1902- \\
1953\end{array}$ & 10,309 & 10,309 & 9,784 & 8,650 \\
\hline $\begin{array}{l}\text { United } \\
\text { Kingdom }\end{array}$ & $\begin{array}{l}\text { National Heights and Weights Survey } \\
\text { (Office of Population Censuses and } \\
\text { Surveys 1985) }\end{array}$ & 1980 & 51.3 & $18-79$ & $\begin{array}{c}1901- \\
1964\end{array}$ & 10,363 & 9,377 & - & - \\
\hline $\begin{array}{l}\text { United } \\
\text { Kingdom }\end{array}$ & $\begin{array}{l}\text { International Social Survey Program } \\
\text { (ISSP Research Group 1988) }\end{array}$ & 1986 & 53.0 & $18-85$ & $\begin{array}{c}1901- \\
1968\end{array}$ & 1,416 & 1,395 & - & - \\
\hline $\begin{array}{l}\text { United } \\
\text { Kingdom }\end{array}$ & $\begin{array}{l}\text { UK National Survey of Sexual } \\
\text { Attitudes and Life Style (Field et al. } \\
1995 \text { ) } \\
\text { UK National Survey of Sexual }\end{array}$ & 1990 & 56.6 & $18-59$ & $\begin{array}{c}1931- \\
1972\end{array}$ & 4,548 & 4,371 & - & - \\
\hline $\begin{array}{l}\text { United } \\
\text { Kingdom }\end{array}$ & $\begin{array}{l}\text { Attitudes and Life Style (National } \\
\text { Centre for Social Research et al. } \\
\text { 2005) }\end{array}$ & 2000 & 57.3 & $18-44$ & $\begin{array}{c}1955- \\
1983\end{array}$ & 12,110 & 11,427 & - & - \\
\hline $\begin{array}{l}\text { United } \\
\text { Kingdom }\end{array}$ & $\begin{array}{l}\text { UK Household Longitudinal Study } \\
\text { (University of Essex, Institute for } \\
\text { Social and Economic Research, and } \\
\text { NatCen Social Research 2016) }\end{array}$ & 2009 & 57.9 & $18-58$ & $\begin{array}{c}1951- \\
1990\end{array}$ & 7,304 & 6,860 & 6,860 & 6,667 \\
\hline $\begin{array}{l}\text { United } \\
\text { Kingdom }\end{array}$ & $\begin{array}{l}\text { UK National Survey of Sexual } \\
\text { Attitudes and Life Style (Johnson et } \\
\text { al. 2015) }\end{array}$ & 2010 & 58.5 & $19-74$ & $\begin{array}{c}1935- \\
1990\end{array}$ & 15,162 & 13,035 & - & - \\
\hline $\begin{array}{l}\text { West } \\
\text { Germany }\end{array}$ & $\begin{array}{l}\text { International Social Survey Program } \\
\text { (ISSP Research Group 1988) }\end{array}$ & 1986 & 53.6 & $18-85$ & $\begin{array}{c}1901- \\
1968\end{array}$ & 2,809 & 2,616 & - & - \\
\hline $\begin{array}{l}\text { West } \\
\text { Germany }\end{array}$ & $\begin{array}{l}\text { German Socio-Economic Panel } \\
\text { (Schupp et al. 2017) }\end{array}$ & $\begin{array}{c}1990- \\
2015\end{array}$ & 52.2 & 18-99 & $\begin{array}{c}1901- \\
1990\end{array}$ & 61,140 & 36,108 & 32,507 & 30,120 \\
\hline $\begin{array}{l}\text { West } \\
\text { Germany }\end{array}$ & $\begin{array}{l}\text { Generations and Gender Program } \\
\text { (Vikat et al. 2007) }\end{array}$ & 2005 & 53.5 & $18-80$ & $\begin{array}{c}1925- \\
1987\end{array}$ & 7,760 & 7,613 & 7,395 & 7,366 \\
\hline
\end{tabular}


Table 2: Observations by birth cohort and country from the International Sibsize and Educational Attainment Database (ISEAD, Monden et al. 2018)

\begin{tabular}{|c|c|c|c|c|c|c|c|c|c|c|}
\hline & $\begin{array}{c}1901- \\
1910\end{array}$ & $\begin{array}{c}1911- \\
1920\end{array}$ & $\begin{array}{c}1921- \\
1930\end{array}$ & $\begin{array}{c}1931- \\
1940\end{array}$ & $\begin{array}{c}1941- \\
1950\end{array}$ & $\begin{array}{c}1951- \\
1960\end{array}$ & $\begin{array}{c}1961- \\
1970\end{array}$ & $\begin{array}{c}1971- \\
1980\end{array}$ & $\begin{array}{c}1981- \\
1990\end{array}$ & Total \\
\hline Australia & & & 873 & 1,042 & 1,443 & 1,931 & 1,882 & 1,165 & 691 & 9,027 \\
\hline Belgium & & & & 756 & 1,065 & 1,405 & 1,508 & 1,226 & 1,059 & 7,019 \\
\hline Bulgaria & & & 1,072 & 2,212 & 2,699 & 2,677 & 3,776 & 2,966 & 1,456 & 16,858 \\
\hline Canada & & 1,784 & 3,430 & 4,156 & 6,100 & 7,786 & 7,719 & 4,696 & 640 & 36,311 \\
\hline China & & & & 2,645 & 5,389 & 7,419 & 8,842 & 6,631 & 5,102 & 36,028 \\
\hline Taiwan & & & 597 & 1,231 & 1,905 & 3,601 & 3,108 & 2,072 & 1,302 & 13,816 \\
\hline Czechia & & & 1,504 & 2,114 & 2,885 & 2,927 & 2,627 & 2,204 & 1,257 & 15,518 \\
\hline Estonia & & & 549 & 1,184 & 1,252 & 1,465 & 1,403 & 1,437 & & 7,290 \\
\hline France & & 1,399 & 5,877 & 13,122 & 22,579 & 26,060 & 22,156 & 10,370 & 4,193 & 105,756 \\
\hline Georgia & & & & 1,280 & 1,200 & 1,903 & 2,022 & 1,830 & 1,312 & 9,547 \\
\hline East Germany & & & 509 & 1,458 & 1,568 & 2,083 & 2,291 & 1,894 & 1,193 & 10,996 \\
\hline West Germany & & 539 & 2,369 & 5,010 & 6,147 & 7,709 & 10,790 & 8,516 & 5,257 & 46,337 \\
\hline Hungary & & & 1,522 & 2,746 & 3,604 & 4,272 & 3,276 & 3,303 & 826 & 19,549 \\
\hline Italy & & & & 595 & 2,221 & 2,199 & 2,467 & 1,519 & 569 & 9,570 \\
\hline Japan & & & 2,739 & 7,284 & 10,732 & 9,819 & 9,688 & 9,363 & 2,914 & 52,539 \\
\hline South Korea & & & & 696 & 1,654 & 2,732 & 3,349 & 2,432 & 1,404 & 12,267 \\
\hline Lithuania & & & & 1,547 & 1,413 & 1,712 & 1,798 & 1,596 & 1,477 & 9,543 \\
\hline Netherlands & & & 724 & 1,939 & 2,983 & 3,679 & 3,948 & 2,081 & & 15,354 \\
\hline Norway & & & & 1,557 & 2,500 & 2,510 & 2,945 & 2,543 & 1,821 & 13,876 \\
\hline Poland & & & 1,238 & 4,301 & 5,475 & 6,778 & 4,579 & 4,390 & 2,860 & 29,621 \\
\hline Romania & & & 695 & 1,946 & 2,085 & 2,368 & 2,264 & 1,822 & 806 & 11,986 \\
\hline Russia & & & 1,558 & 2,710 & 2,575 & 3,807 & 3,310 & 2,398 & 943 & 17,301 \\
\hline Spain & & & & 495 & 739 & 815 & 1,051 & 1,186 & 985 & 5,271 \\
\hline Sweden & & & & 767 & 1,729 & 1,633 & 1,783 & 1,599 & 1,453 & 8,964 \\
\hline United Kingdom & 813 & 3,198 & 4,394 & 5,668 & 7,874 & 9,469 & 10,736 & 8,334 & 6,288 & 56,774 \\
\hline USA & 2,336 & 5,361 & 7,275 & 6,963 & 10,224 & 11,984 & 8,352 & 4,750 & 2,091 & 59,336 \\
\hline Total & 3,149 & 12,281 & 36,925 & 75,424 & 110,040 & 130,743 & 127,670 & 92,323 & 47,899 & 636,454 \\
\hline
\end{tabular}

'Sibship size' is the total number of brothers and sisters a respondent has ever had, including the respondents themselves. Many surveys did not specify whether respondents should count half- or nonbiological siblings such as stepsiblings and adopted siblings, while others clarified that siblings should include the number of full, half, and nonbiological siblings. Since we did not identify any single survey that asked for the number of biological siblings specifically, we assumed that all values of the number of siblings represented sibship size inclusive of all types of siblings. To 
minimize an arbitrary variation in the upper bound of sibship size across surveys, we use the value 11 for all sibship size values higher than 10 .

'Parental education' is measured as a binary variable, indicating whether at least one parent holds a tertiary degree. This variable usually refers to the persons the respondent identifies as their father and mother; most surveys do not specify who this should be, but it is most likely the social parent the respondent grew up with. We converted the original measurements of parental education into the ISCED classification (with ISCED groups 5 and 6 indicating tertiary education, UNESCO 2006) and relied on the so-called 'dominance' approach (Erikson 1984) for creating our final variable, i.e., substituting father's education with mother's education if father's education is missing or lower than mother's education. We draw on parental education - rather than occupation, income, or social class - as a measure of socioeconomic status, for several reasons (Präg and Subramanian 2017). First, education reflects both parental material and nonmaterial resources and social status in a broad fashion. Second, the ISCED, with its high degree of cross-national standardization, allows meaningful comparison of educational groups across countries. Third, educational attainment is usually completed in early adulthood and remains for the most part stable across the life course, unlike income or occupation. Fourth, compared to income, which usually comes with a large proportion of nonrespondents, education is an easy-tomeasure indicator of socioeconomic status. Finally, education is a meaningful measure of the socioeconomic status of both men and women and those outside the labor force.

'Only-children' are respondents who report not having any siblings. Respondents are considered to be from 'large families' when they report having four or more siblings, i.e., report a sibship size of five and more.

\subsection{Assessment of trends over time}

Divergence or convergence of demographic disparities by parental background is calculated separately for each country as a simple ordinary least squares regression model. For each country and birth year of respondents, we calculate the disparity between parents with and without a degree and regress this disparity on the respondents' birth years:

$$
M_{\text {loeduc }}-M_{\text {hieduc }}=a+b \times X_{\text {birthyear }}+\epsilon
$$

where $M_{\text {loeduc }}$ and $M_{\text {hieduc }}$ are the average sibship sizes (or the prevalence of onlychildren or large families) per country and birth year for higher- and lower-educated parents. The coefficient $b$ (as shown in Figures 4, 6, 8, and 11) indicates the trend of the 
gap in sibship size between the groups of children with different parental education levels - convergence when negative or divergence when positive. For instance, for the analysis of average sibship sizes, $b$ can thus be interpreted as the annual rate of change in the average gap between parents with a tertiary degree and parents without such a degree.

To address possible nonlinearities in the trends over time, we also fitted a second model where we added the quadratic term $c \times X_{\text {birthyear }}^{2}$. Countries with (inverted) Ushaped developments (i.e., where the coefficient $c$ was significantly different from 0 ) were excluded from further analyses.

\subsection{Replicability}

The data used for our analyses is publicly available. Details about the data used can be found in Monden et al. (2018). We provide a set of Stata and R files on-line for replicating the analyses presented in this manuscript (Präg, Choi, and Monden 2020).

\section{Results}

\subsection{Average sibship size, prevalence of only-children, and prevalence of large families over time}

Figure 1 presents the average sibship size over time for 26 countries. Across the board, average sibship sizes have decreased for birth cohorts over the $20^{\text {th }}$ century. Reductions have often been considerable; for instance, from almost 6 in the oldest birth cohort in Canada to around 2 in the most recent birth cohort. But this pattern is not without exceptions: In Germany the average sibship size remained relatively stable over the observation period.

Figure 2 shows the prevalence of only-children and large families (i.e., sibship sizes of five and above) over time for 26 countries. There has been a decrease in large families in all countries and, similar to average sibship size, the reduction has often been large. For instance, in the United States the percentage of respondents from large families has dropped from more than $60 \%$ for those born at the beginning of the last century to about $30 \%$ for those born at the end. For only-children, change has been more modest. Apart from China with its marked increase in only-children, from 13\% in the 1930 s to almost $50 \%$ in the 1980 s, few countries show strong variation over time. 


\subsection{Disparities in sibship size}

Figure 3 stratifies average sibsize by parental education. In all countries, children of parents without a tertiary degree have on average more siblings than children of parents with a tertiary degree. The size of this difference varies over time. For instance, in the most recent US birth cohort we find 4.4 siblings in lower-educated families and 3.6 siblings in higher-educated families, a difference of almost an entire child. In Norway, however, the sibship size is on average 2.9 in families with higher-educated parents and 3.0 among their lower-educated counterparts - a miniscule difference. In some cases, such as China, Romania, and Spain, confidence intervals overlap at some time points, indicating that the null hypothesis of no difference between higher- and lower-educated parents cannot be rejected at conventional levels of statistical precision. The gaps tend to be greater in some countries than in others. In Eastern European countries the gap in sibship size between those with high-educated parents and those with low-educated parents is relatively large. Another noticeable pattern in a majority of countries is a narrowing trend of disparity by parental educational degree. Only a few countries show a widening gap. We further observe a two-phase pattern in a number of countries, such as Italy and the United Kingdom, where convergence took place between birth cohorts before the 1940s and has been followed by a period of relative stability. Further comparisons by region show no discernible patterns. 
Figure 1: Average sibship size in 26 countries, 1901-1990 birth cohorts, $N=636,454$. Error bands denote $95 \%$ confidence intervals.

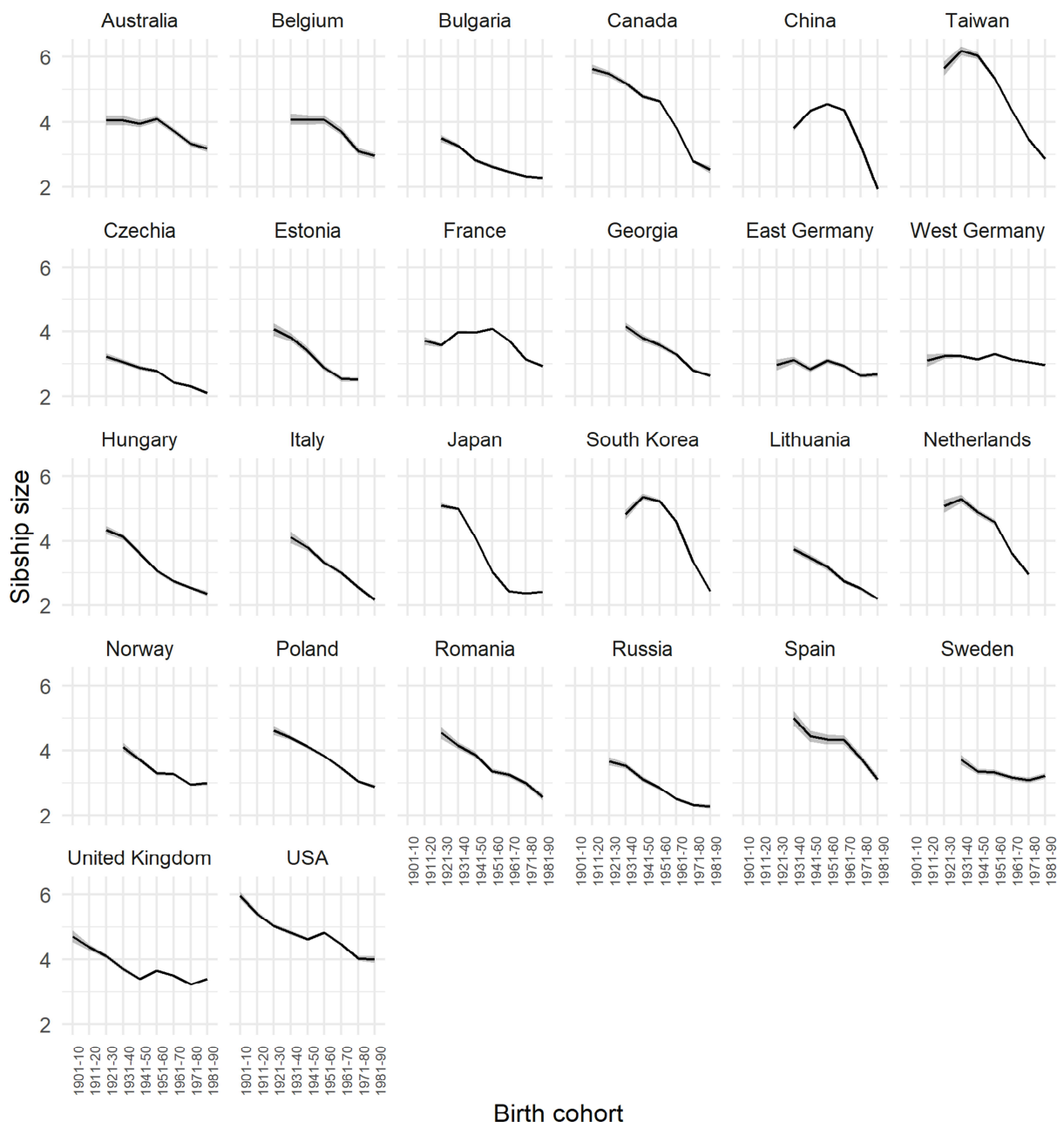

Source: ISEAD (Monden et al. 2018), own calculations. 
Präg, Choi \& Monden: The sibsize revolution in international context

Figure 2: Prevalence of only-children and large families (sibships of 5 and more) in 26 countries, 1901-1990 birth cohorts, $N=636,454$. Error bands denote $95 \%$ confidence intervals.

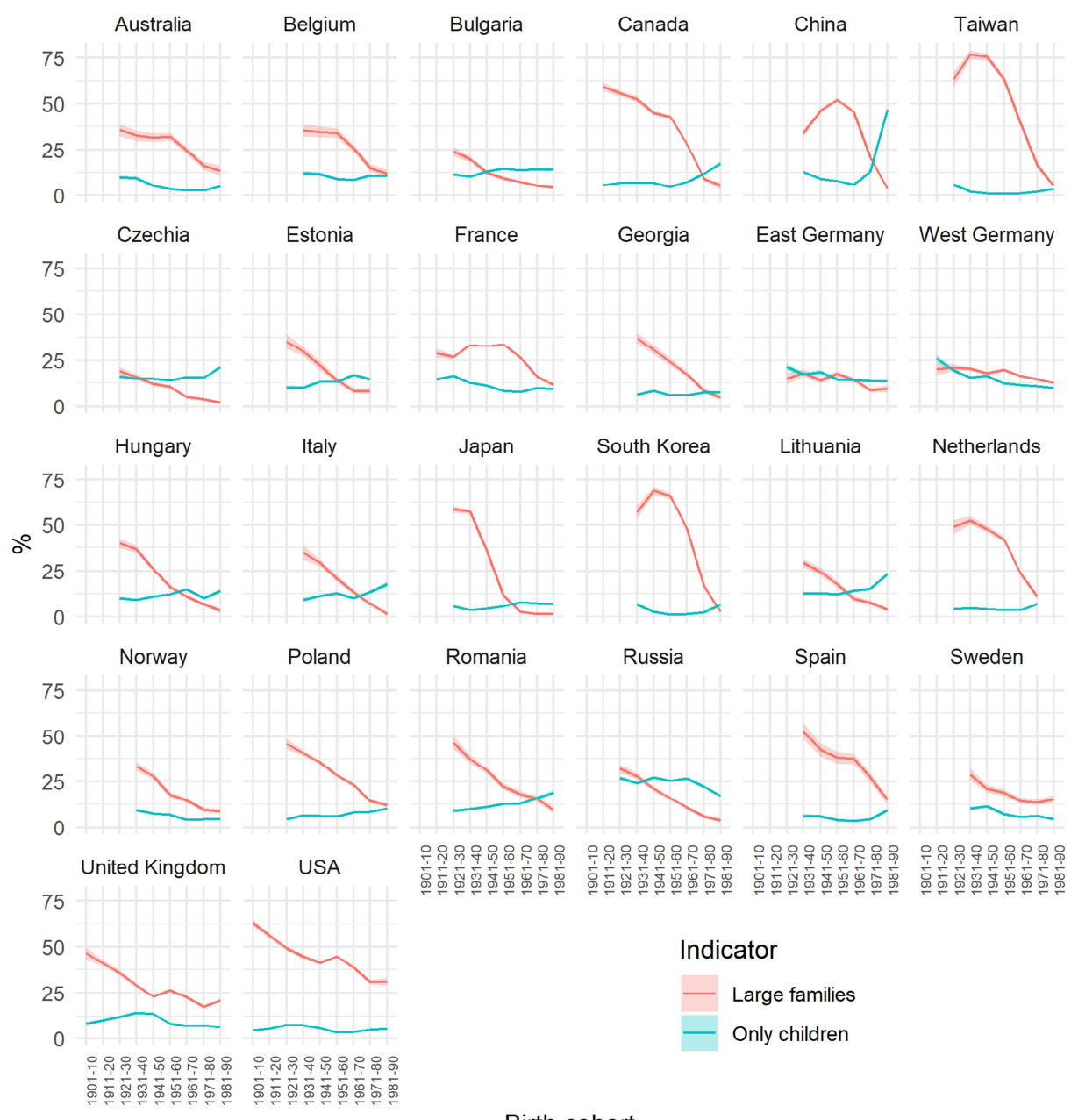

Birth cohort

Source: ISEAD (Monden et al. 2018), own calculations. 
Figure 3: Sibship size by parental education in 26 countries, 1901-1990 birth cohorts, $N=\mathbf{5 3 7 , 8 0 7}$. Error bands denote $95 \%$ confidence intervals.

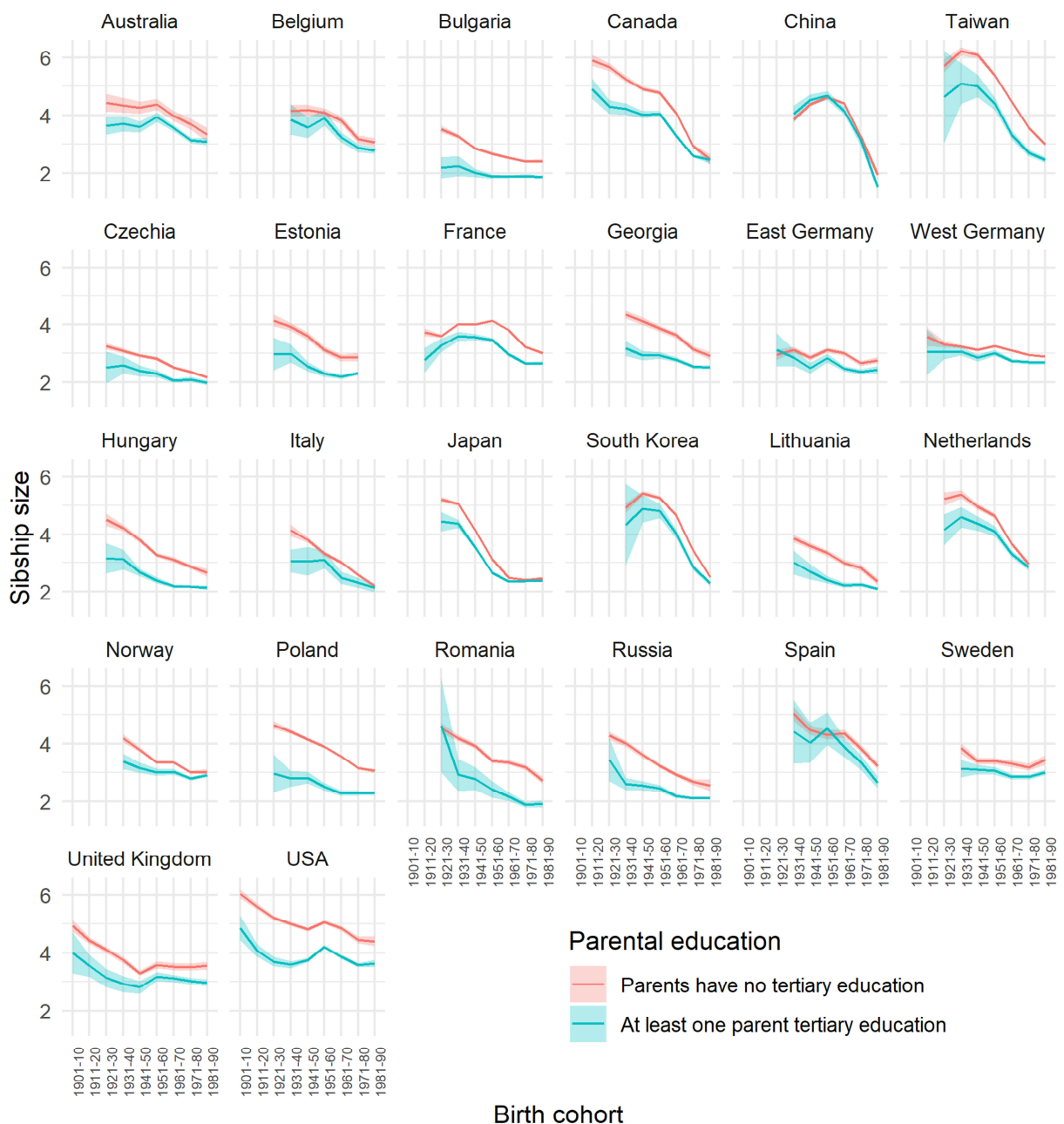

Source: ISEAD (Monden et al. 2018), own calculations.

Figure 4 assesses the convergence between higher- and lower-educated families over time more formally. The trend coefficient indicates whether the gap between higher- and lower-educated families closes over time, indicating convergence, or 
widens, indicating divergence. For instance, in Poland the average sibship size gap between children of higher- and lower-educated parents reduced by 0.02 per year, which equates to one child over a fifty-year period. Figure 4 confirms that virtually all countries have experienced a convergence in sibship sizes. We detect a diverging trend in only two countries, France and Romania, but neither of these are statistically significant at the conventional level.

\section{Figure 4: Convergence and divergence of large family prevalence by parental education in 19 countries, 1901-1990 birth cohorts. Countries with nonlinear trends excluded. Error bars indicate $95 \%$ confidence intervals.}

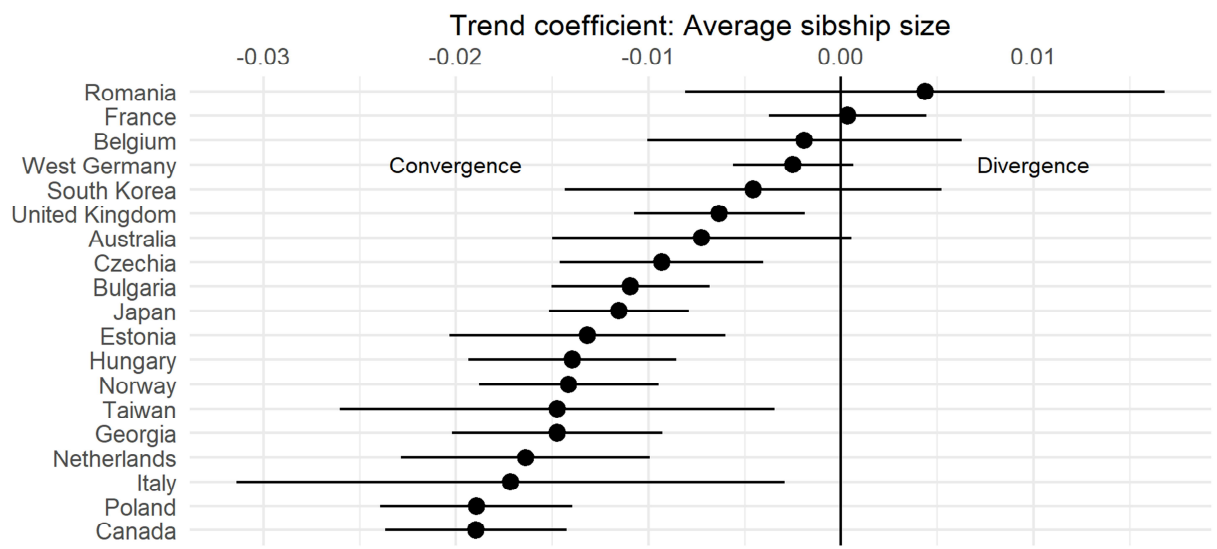

Source: ISEAD (Monden et al. 2018), own calculations.

In sum, our analyses show that children of lower-educated parents grow up with more siblings than those of higher-educated parents, but that, this disparity has declined over time in virtually all countries in our study.

\subsection{Disparities in growing up as an only child}

Figure 5 shows the prevalence of only-children by parental education. First, there is less social disparity when it comes to only-children. In more than half of the countries there is no difference between lower- and higher-educated parents in the prevalence of onlychildren. Second, however, we find an important regional difference in the countries under study. In the Eastern European countries - Bulgaria, Estonia, Hungary, Poland, 
Romania, and Russia - there is a marked social disparity in the prevalence of onlychildren. In these countries, prevalence rates are higher among the higher-educated. For instance, in Poland the prevalence rate of only-children in the latest cohort is $7.8 \%$ among those with lower-educated parents and $17.2 \%$ among those with higher-educated parents. Third, there is no clear trend in the changes in the disparity (Figure 6). The majority of countries show a widening gap, but most of the growth is not statistically significant - except in the United States, Canada, Georgia, and Estonia.

\subsection{Disparities in growing up in large families}

Figure 7 shows the percentage of children growing up in a large family by parental education. Large families are those with five children or more. First, we see a declining trend for large family prevalence over time. For instance, in Georgia the prevalence of children from large families fell from $41.1 \%$ among the lower educated in the 1930 s to $7.8 \%$ in the $1980 \mathrm{~s}$, the latest cohort, and for the higher educated from $19.2 \%$ to $3.8 \%$ over the same period of time, a decrease of more than three-quarters.

Second, we see that across all countries, children of lower-educated parents are more likely to live in large families, with differences in prevalence rates often around $10 \%$. For instance, in the youngest US cohort (1981-1990), 24.6\% of respondents from higher-educated parents and $38.6 \%$ of respondents from lower-educated parents grew up in large families.

Third, we see a general trend towards convergence of social disparities over the course of the $20^{\text {th }}$ century. Figure 8 reveals that eleven countries show a clear trend towards convergence. Three countries (West Germany, Romania, and Belgium) show a trend towards divergence, but do not reach the conventional levels of statistical significance. Seven countries, which are not shown in Figure 8 because of their nonlinear trends (East Germany, Italy, Lithuania, Spain, Sweden, the United Kingdom, and the USA), mostly indicate stability over the course of the $20^{\text {th }}$ century, according to Figure 7. In terms of regional variation, no clear patterns arise. 
Präg, Choi \& Monden: The sibsize revolution in international context

Figure 5: Only-children by parental education in 26 countries, 1901-1990 birth cohorts, $N=\mathbf{5 3 7 , 8 0 7}$. Error bands denote $95 \%$ confidence intervals. China plotted on a different $y$-axis scale to facilitate comparison with other countries.

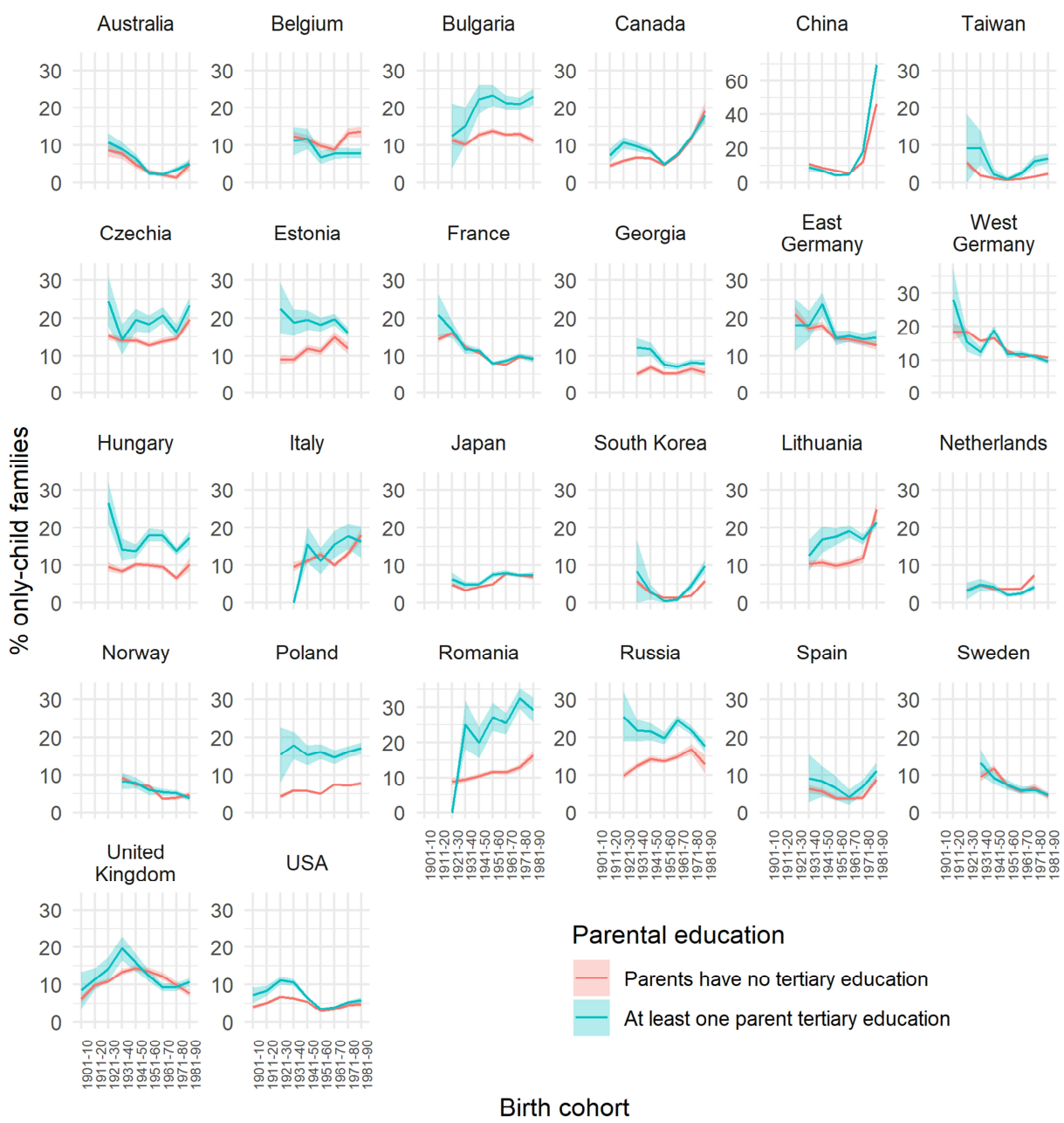

Source: ISEAD (Monden et al. 2018), own calculations. 
Figure 6: Convergence and divergence of only-children prevalence by parental education in 23 countries, 1901-1990 birth cohorts. Countries with nonlinear trends excluded. Error bars indicate $95 \%$ confidence intervals.

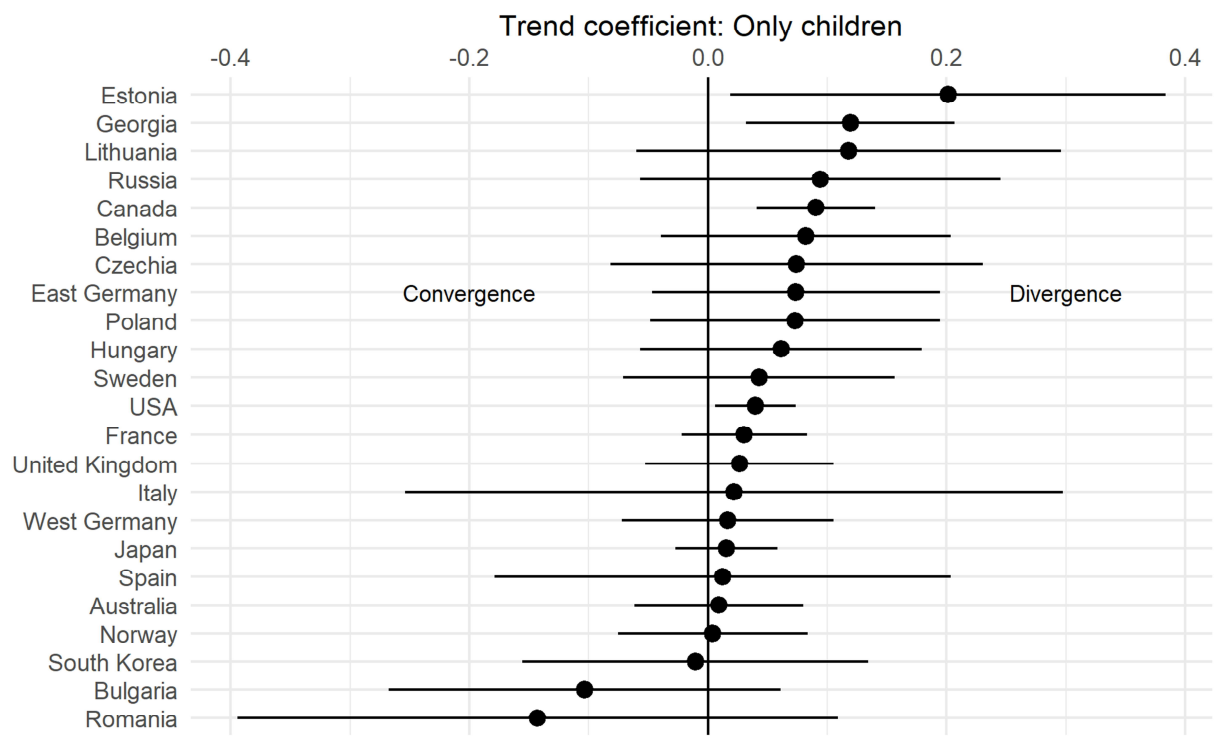

Source: ISEAD (Monden et al. 2018), own calculations. 
Präg, Choi \& Monden: The sibsize revolution in international context

Figure 7: Large families by parental education in 26 countries, 1901-1990 birth cohorts, $N=\mathbf{5 3 7 , 8 0 7}$. Error bands denote $95 \%$ confidence intervals.

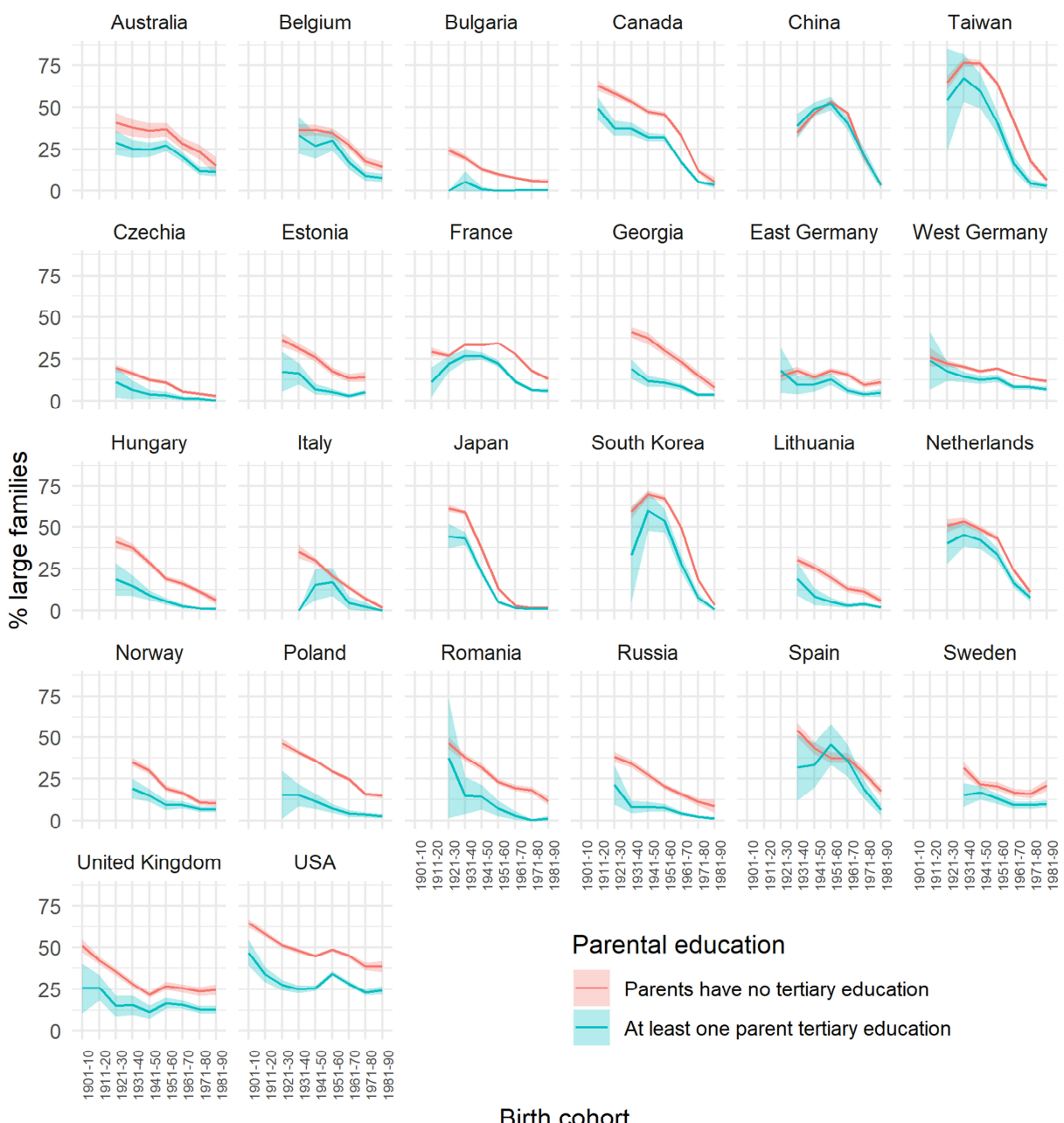

Source: ISEAD (Monden et al. 2018), own calculations. 
Figure 8: Convergence and divergence of large family prevalence by parental education in 19 countries, 1901-1990 birth cohorts. Countries with nonlinear trends excluded. Error bars indicate $95 \%$ confidence intervals.

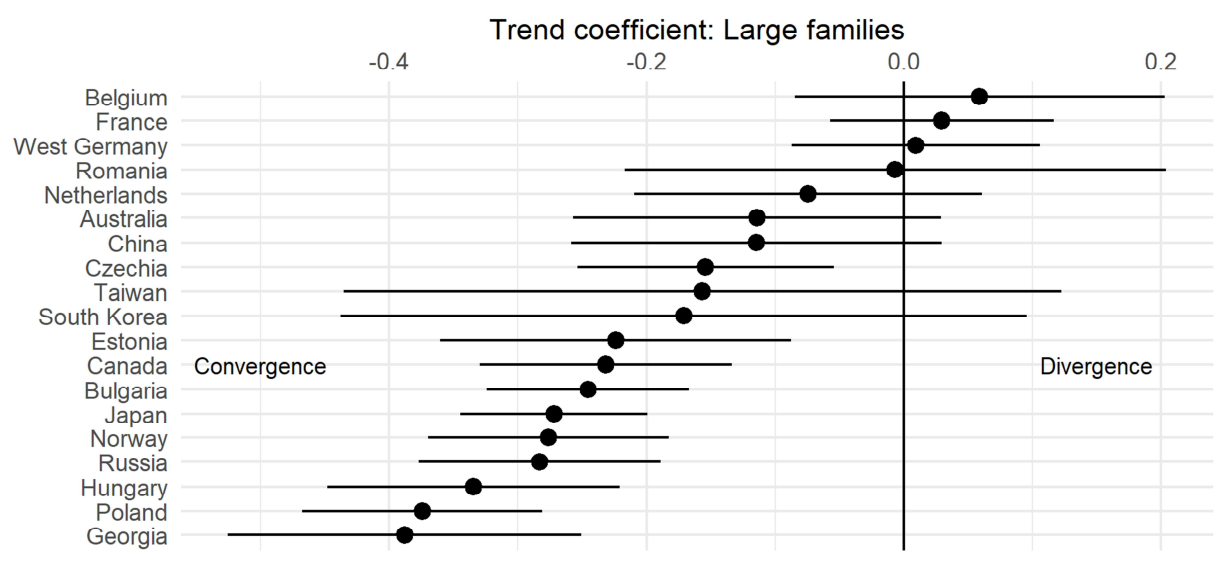

Source: ISEAD (Monden et al. 2018), own calculations.

\subsection{Disparities in sibship size, distinguishing between paternal and maternal education}

Jalovaara et al. (2019) find important gender differences in educational differentials for cohort fertility and childlessness in Nordic countries. Figures 9 and 10 stratify trends in average sibship size by maternal and paternal education. The pattern here is very similar to that shown in Figure 3, where parental education was determined according to the dominance principle. Given that men on average had higher educational attainment across much of the $20^{\text {th }}$ century (less so in recent cohorts), the similarity between Figures 3 and 10 is not surprising. Regarding mother's education, we can see that disparities and changes are more moderate for maternal education (Figure 9) than for paternal education in Figure 10 (or 3, for that matter). This is also to be expected because disparities should be more moderate in mother's education than in father's education. There is homogamy (the correlation between father's education and mother's education), but such a homogamous tendency is not perfect (that is, the correlation is never close to 1), so we can expect Figures 9 and 10 to show similar patterns but Figure 9 to show much more moderate trends. 
Präg, Choi \& Monden: The sibsize revolution in international context

Figure 9: Sibship size by maternal education in 26 countries, 1901-1990 birth cohorts, $N=\mathbf{5 1 9 , 7 3 1}$. Error bands denote $95 \%$ confidence intervals.
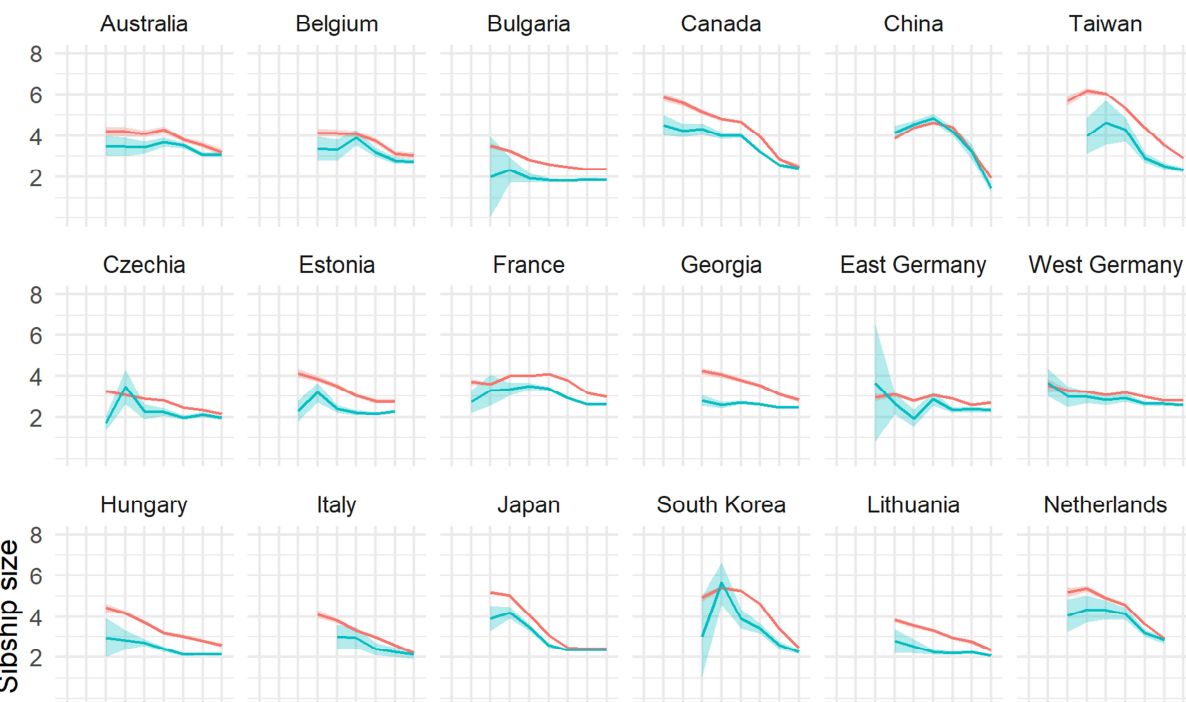

West Germany
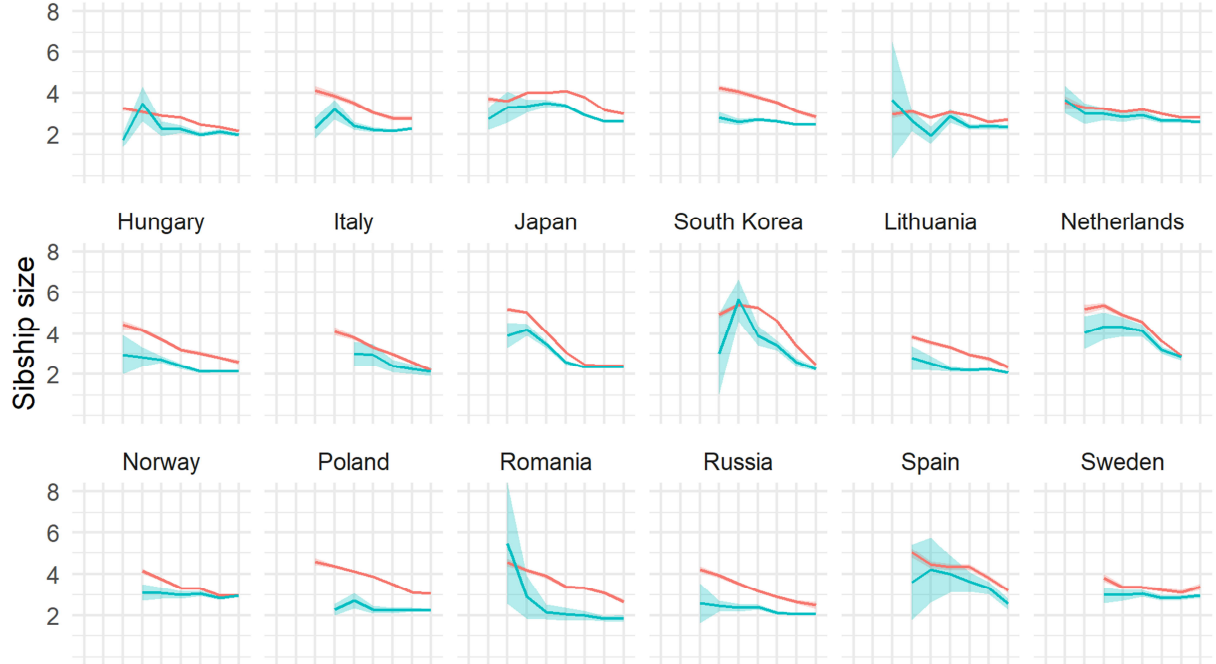

Netherlands

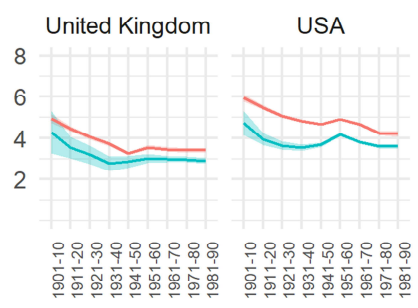

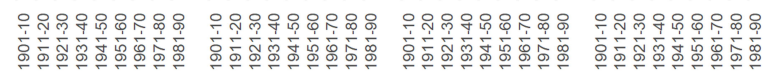

Mother's education

No tertiary education

Tertiary education

Birth cohort

Source: ISEAD (Monden et al. 2018), own calculations. 
Figure 10: Development of sibship size by paternal education in 26 countries, 1901-90 birth cohorts, $N=495,848$. Error bands indicate $95 \%$ confidence intervals.

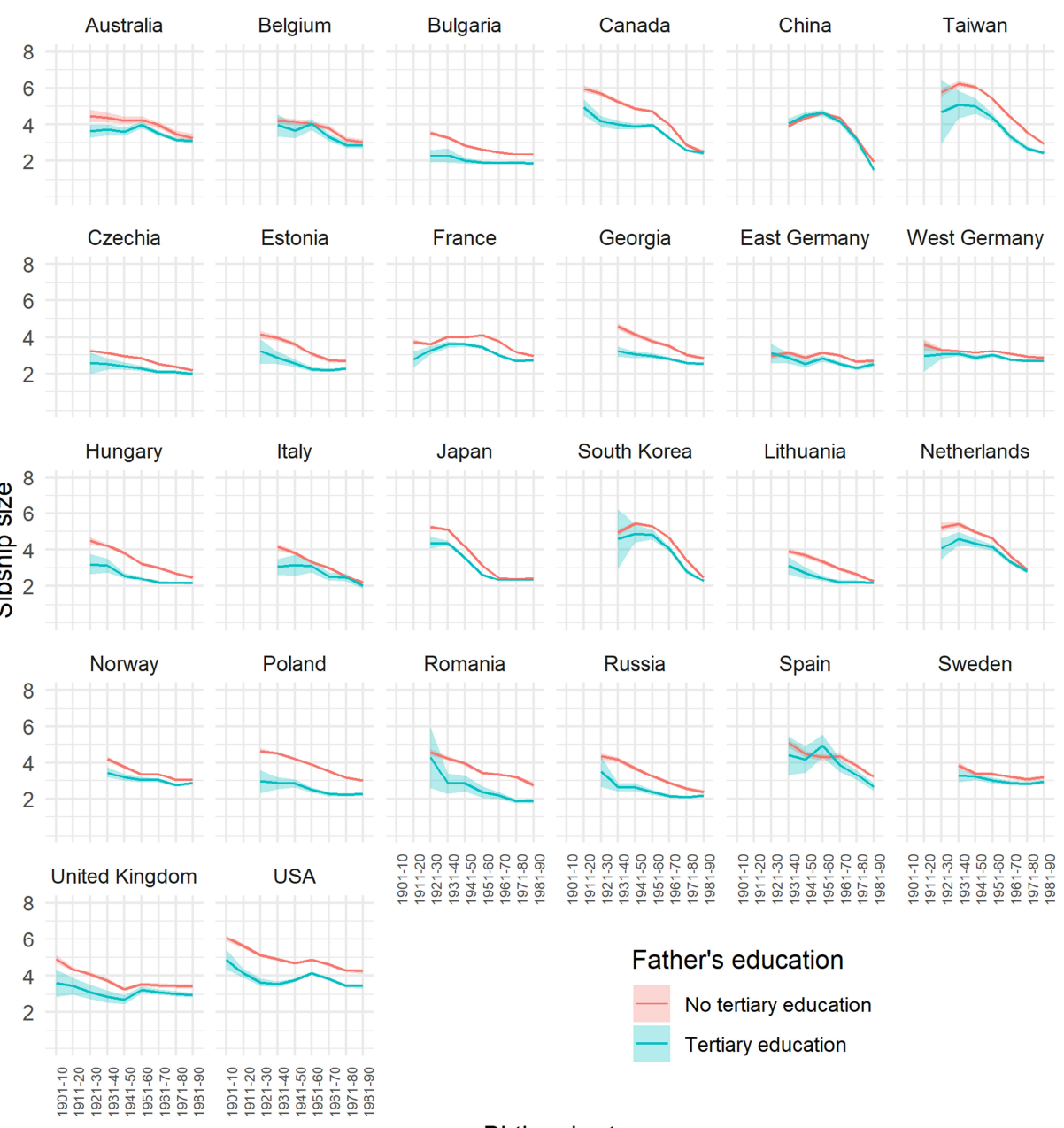

Birth cohort

Source: ISEAD (Monden et al. 2018), own calculations. 
Figure 11 compares the overall trend over time in average sibship size by paternal and maternal education in a formal manner, showing that $95 \%$ confidence intervals overlap in all countries where both men and women follow linear trends.

Figure 11: Convergence and divergence of sibship size disparity by paternal and maternal education in 25 countries, 1901-90 birth cohorts. Only linear estimates are shown. Error bars indicate $95 \%$ confidence intervals.

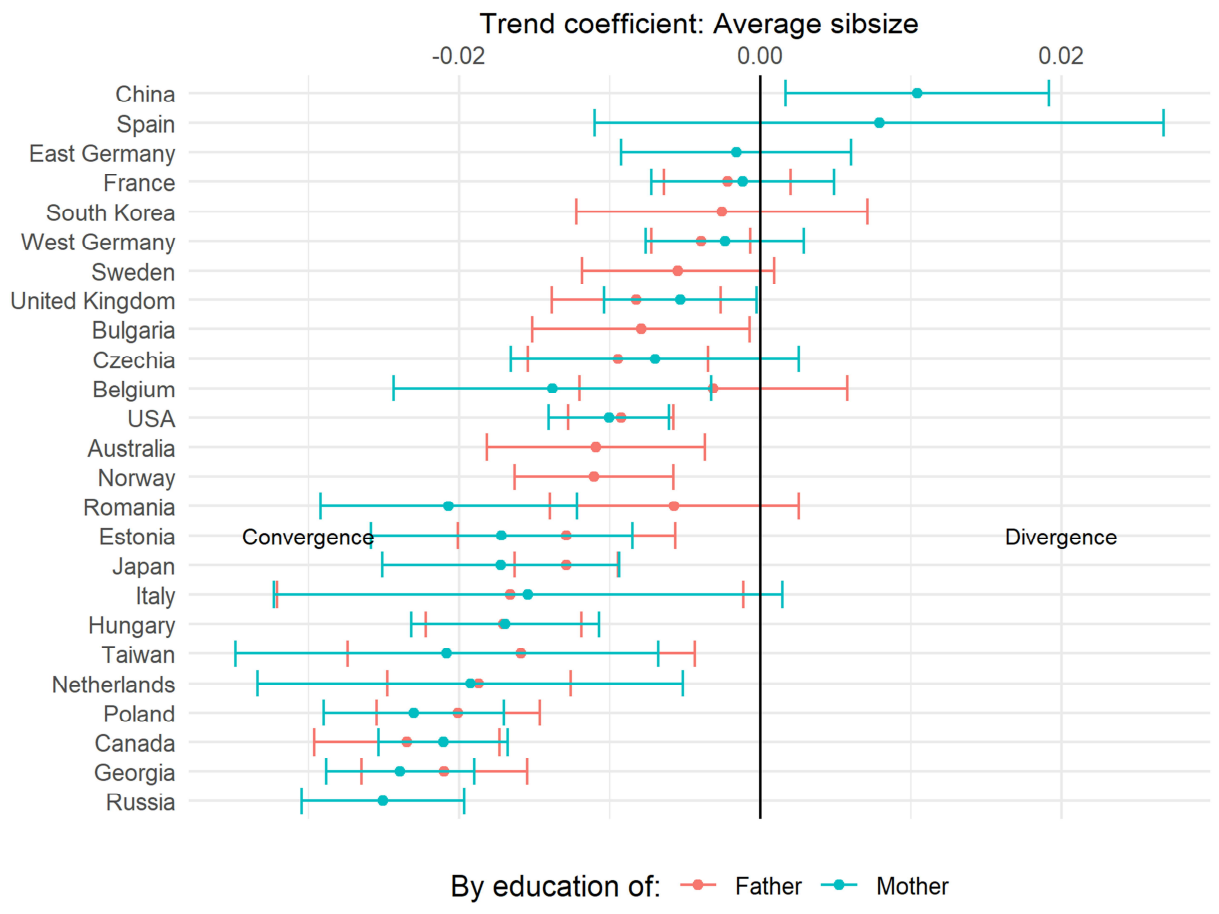

Source: ISEAD (Monden et al. 2018), own calculations.

\section{Summary and discussion}

Our analysis of sibship sizes in 9 birth cohorts across the $20^{\text {th }}$ century and in 26 lowfertility countries shows that sibship size has declined in virtually all of these 26 
countries. As sibship size is an important factor affecting children's early and later life outcomes, this finding sends a positive message of declining social inequalities stemming from sibsize effects. As was expected, this was largely due to a uniform and often strong trend towards fewer large families. Regarding only-children the trends are less pronounced, with disparities being largely stable in most countries.

Social disparities in sibship size are becoming smaller. Sibship size is socially stratified: Children of parents with lower education grow up in larger families than children of higher-educated parents. In our study this holds true across virtually all countries and all points in in time. However, the differences are becoming smaller. Fahey (2017) argues that the convergence of sibship sizes in the United States is an oftoverlooked social trend. We find support for Fahey's (2017) optimistic notion of a 'sibsize revolution' in the low-fertility countries we selected for this study. The vast majority of countries show a converging trend between the number of children of higher- and lower-educated parents, suggesting that any negative sibling effects are occurring less often these days. Furthermore, in most countries the decline is greater than in the United States, suggesting that Fahey's (2017) finding based on US data shows the lower bound of the 'sibsize revolution.' This suggests that the evolution of family size is a largely overlooked but nonetheless key driver of the improvement in the intergenerational path of inequality not only in the United States but also in many other countries, especially those having undergone the transition to low fertility. Choi et al. (2020) suggest that the negative effect on a child's education of additional siblings has increased over time, yet our analyses suggest that such an effect might be mitigated by demographic processes: Fewer children experience a larger number of siblings, and the number of siblings is less socially stratified than before.

The convergence in sibship sizes is taking place at the top of the sibship size distribution and not at the bottom. We observe a general decline in the prevalence of large families over the course of the century (with sibship sizes of five and above considered to be large families). While children of lower-educated parents are more likely to grow up in larger families than those with higher-educated parents, this gap is closing over time in many countries under study. We find little convergence at the bottom of the sibship size distribution. The prevalence of only-children is largely stable over time, and social disparities in raising only-children only arise in Eastern European countries.

Decreasing sibship sizes can have important implications for public policies and public spending. For example, measures such as child benefits and higher public expenditure on education and families reduce the gap in educational achievement between children from small and large families (Park 2008). However, our results show that policy measures should not be designed on the basis of total fertility rates when what really is matters is the size of the family a child grows up in. The recent trend 
towards increasing childlessness (Sobotka 2017) poses a challenge for policies that assume, based on the total fertility rate, that family size is decreasing. The implications for our thinking about child benefits, for instance, would be quite different. Lam and Marteleto (2008) argue that cohort size and sibship size - which are important because children compete for resources both in the family and at the population level - can even move in opposite directions.

Research on intergenerational social mobility has long been at the forefront of cross-nationally comparative research, assembling large data sets from many societies to examine parent-offspring correlations in socioeconomic status across many societies (Featherman, Jones, and Hauser 1975; Lipset and Bendix 1959). Current efforts to advance intergenerational social mobility research by linking it more strongly with demographic processes focus on single country cases (Breen and Ermisch 2017; Breen and Salazar 2011; Mare 2011; Mare and Maralani 2006; Song and Campbell 2017). Our study takes this line of research back to a cross-national perspective. Given the potential importance of demographic transitions for the reproduction of social inequalities, this country-comparative approach is an important piece of the puzzle. While our findings show that there is great universality in the trend towards smaller sibship sizes and declining social disparities in sibship size, we also find important country variance in these trends.

We have to acknowledge a number of limitations to our analyses. First, the survey data we used usually did not allow us to assess the family structure of siblings. For instance, it would be interesting to take into account the time that siblings have lived together and to distinguish between full siblings, half-siblings, and adopted siblings, but this is not possible across such a large number of data sets, cohorts, and countries. This problem particularly applies to the most recent cohorts, where families have grown more diverse and complex (Brown, Manning, and Stykes 2015). Blake (1981) finds that sibship size is correlated with family structure in the United States, such that non-intact families are more likely to have small family size. If this holds in many countries other than the United States, the growing instability of family structures may offset the potential benefits of being brought up in small families, and therefore the extent of the 'sibsize revolution' might be exaggerated.

Second, due to the inclusion of relatively old respondents, our sample might be affected by differential mortality. However, only $3.5 \%$ of the respondents are older than 75 years of age, and 12\% are older than 65 . Moreover, from the 1931-1940 birth cohort onwards there are very few respondents over age $65(7 \%)$, going down to $1.5 \%$ in the $1941+$ birth cohorts. Given differences in mortality and survivorship across socioeconomic statuses, our disparity estimates should be considered lower-bounds estimates, especially for older birth cohorts. This means changing trends could be underestimated. However, this possibility corroborates, rather than undermines, our 
main result of declining disparity. Third, throughout our analyses we used the same indicator of parental education across the birth cohorts, distinguishing between those with at least one tertiary degree and those with none. Given the educational expansion that occurred during the $20^{\text {th }}$ century, the composition of groups of individuals obtaining a degree changes over time. An alternative approach would be to use a relative measure of education for comparisons over time. However, this turned out not to be feasible, as education does not follow a well-behaved normal distribution, particularly over a long period of time and multiple countries, thus sometimes leaving created cells empty.

The first two limitations are due to the nature of the data we used. Detailed data that links individuals across generations and by relationship is only available for a very small number of countries, and even then we would not be able to look across many birth cohorts. Given the importance of family for children, it is surprising that simple statistics on children's family circumstances are not systematically available from national statistical offices or similar agencies. It is high time we start producing indicators that directly measure the family circumstances that children grow up in. Analysis of large-scale survey data is helpful in studying trends, but we need more recent and more reliable data, ideally linkable to administrative data, to make further progress.

As we have argued before, sibship size is a linchpin in the intergenerational transmission of resources. Given the decline in average sibship sizes over time and the convergence of social disparities in sibship size, our findings support an optimistic view of reduced social disparities and increasing intergenerational mobility.

\section{Acknowledgments}

Previous versions of this study were presented at the annual meeting of the Population Association of America in Denver 2018 and the German Society for Demography conference in Cologne 2018. We thank discussants at these meetings as well as the anonymous reviewers of Demographic Research for constructive comments and Riley Taiji, Manting Chen, and Jung In for research assistance. The data used in this study is publicly available (Monden et al. 2018) and replication materials can be found online (Präg, Choi, and Monden 2020). This study has received funding from the European Research Council (ERC) under the European Union's Horizon 2020 research and innovation program under grant agreement no. 681546 (FAMSIZEMATTERS). 


\section{References}

Angrist, J., Lavy, V., and Schlosser, A. (2010). Multiple experiments for the causal link between the quantity and quality of children. Journal of Labor Economics 28(4): 773-824. doi:10.1086/653830.

Balbo, N., Billari, F.C., and Mills, M. (2013). Fertility in advanced societies: A review of research. European Journal of Population 29(1): 1-38. doi:10.1007/s10680012-9277-y.

Björklund, A. and Salvanes, K.G. (2011). Education and family background: Mechanisms and policies. In: Hanushek, E.A., Machin, S., and Woessmann, L. (eds.). Handbook of the economics of education. Amsterdam: Elsevier: 201-247. doi:10.1016/B978-0-444-53429-3.00003-X.

Blaabæk, E.H., Jæger, M.M., and Molitoris, J. (2020). Family size and educational attainment: Cousins, contexts, and compensation. European Journal of Population 36: 575-600. doi:10.1007/s10680-019-09543-y.

Black, S.E., Devereux, P.J., and Salvanes, K.G. (2005). The more the merrier? The effect of family size and birth order on children's education. Quarterly Journal of Economics 120(2): 669-700. doi:10.1093/qje/120.2.669.

Blake, J. (1981). Family size and the quality of children. Demography 18(4): 421-442. doi: $10.2307 / 2060941$.

Blau, P.M. and Duncan, O.D. (1967). The American occupational structure. New York: Wiley.

Breen, R. and Ermisch, J. (2017). Educational reproduction in Great Britain: A prospective approach. European Sociological Review 33(4): 594-603. doi:10.1093/esr/jcx061.

Breen, R. and Salazar, L. (2011). Educational assortative mating and earnings inequality in the United States. American Journal of Sociology 117(3): 808-843. doi:10.1086/661778.

Breen, R., Ermisch, J., and Helske, S. (2019). Educational reproduction in Europe: A descriptive account. Demographic Research 41(49): 1373-1400. doi:10.4054/ DemRes.2019.41.49.

Brown, S.L., Manning, W.D., and Stykes, J.B. (2015). Family structure and child wellbeing: Integrating family complexity. Journal of Marriage and Family 77(1): 177-190. doi:10.1111/jomf.12145. 
Cameron, L., Erkal, N., Gangadharan, L., and Meng, X. (2013). Little Emperors: Behavioral impacts of China's one-child policy. Science 339(6122): 953-957. doi:10.1126/science.1230221.

Chang, Y.-h. (2002). Taiwan Social Change Survey (Round 4, Year 2). Family. Taipei: Academia Sinica, Survey Research Data Archive. doi:10.6141/TW-SRDAC00109\1-1.

Chang, Y.-h. (2014). Taiwan Social Change Survey (Round 4, Year 5). Religion. Taipei: Academia Sinica, Survey Research Data Archive. doi:10.6141/TW-SRDAR090033-1.

Chang, Y.-h. (2016). Taiwan Social Change Survey (Round 6, Year 3). Social stratification. Taipei: Academia Sinica, Survey Research Data Archive. doi:10.6141/TW-SRDA-C00223_1-1.

Chiu, H.-y. (1999). Taiwan Social Change Survey (Round 3, Year 3). Social stratification and social mobility. Taipei: Academia Sinica, Survey Research Data Archive. doi:10.6141/TW-SRDA-C00009\1-1.

Choe, M.K., Tsuya, N., Rindfuss, R., and Bumpass, L.L. (2013). National Survey on Family and Economic Conditions. Ann Arbor: Inter-University Consortium for Political and Social Research. doi:10.3886/ICPSR34647.v1.

Choi, S. and Monden, C. (2017). Where it matters to be the only one: School performance outcomes of only-children across 31 countries. Socarxiv. doi:10.17605/OSF.IO/KC6X5.

Choi, S., Taiji, R., Chen, M., and Monden, C. (2020). Cohort trends in the association between sibship size and educational attainment in 26 low-fertility countries. Demography 57(3): 1035-1062. doi:10.1007/s13524-020-00885-5.

Chu, C.-y. (2002). Panel Study of Family Dynamics. RI1999. Taipei: Academia Sinica, Survey Research Data Archive. doi:10.6141/TW-SRDA-C00102-1.

Cichomski, B., Jerzynski, T., and Zielinski, M. (2007). Polish General Social Survey 1992-2002. Ann Arbor: Inter-University Consortium for Political and Social Research. doi:10.3886/ICPSR20501.v1.

CIS (2013). Prestigio ocupacional y estructura social. Madrid: Centro de Investigaciones Sociolgicas, Estudio 3004.

Clark, G. (2007). A farewell to alms. A brief economic history of the world. Princeton: Princeton University Press. 
Conley, D. and Glauber, R. (2006). Parental educational investment and children's academic risk: Estimates of the impact of sibship size and birth order from exogenous variation in fertility. Journal of Human Resources XLI(4): 722-737. doi:10.3368/jhr.XLI.4.722.

De Graaf, N.D., Graaf, P.M. de, Kraaykamp, G., and Ultee, W. (1998). Family Survey Dutch Population 1998. The Hague: DANS. doi:10.17026/dans-zzu-yw93.

De Graaf, N.D., Graaf, P.M. de, Kraaykamp, G., and Ultee, W. (2000). Family Survey Dutch Population 2000. The Hague: DANS. doi:10.17026/dans-z8n-9wj5.

De Graaf, N.D., Graaf, P.M. de, Kraaykamp, G., and Ultee, W. (2004). Family Survey Dutch Population 2003. The Hague: DANS. doi:10.17026/dans-xm2-5fue.

Downey, D.B., Condron, D.J., and Yucel, D. (2015). Number of siblings and social skills revisited among American fifth graders. Journal of Family Issues 36(2): 273-296. doi:10.1177/0192513X13507569.

Dribe, M., Oris, M., and Pozzi, L. (2014). Socioeconomic status and fertility before, during, and after the demographic transition. Demographic Research S14(7): 161-182. doi:10.4054/DemRes.2014.31.7.

Dunifon, R., Fomby, P., and Musick, K. (2017). Siblings and children's time use in the United States. Demographic Research 37(49): 1611-1624. doi:10.4054/Dem Res.2017.37.49.

Duvander, A.-Z., Lappegård, T., Andersen, S.N., Garðarsdóttir, Ó., Neyer, G., and Viklund, I. (2019). Parental leave policies and continued childbearing in Iceland, Norway, and Sweden. Demographic Research 40(51): 1501-1528. doi:10.4054/ DemRes.2019.40.51.

Erikson, R. (1984). Social class of men, women, and families. Sociology 18(4): 500514. doi: $10.1177 / 0038038584018004003$.

Ernst, C. and Angst, J. (1983). Birth order. Its influence on personality. Berlin: Springer. doi:10.1007/978-3-642-68399-2.

Fahey, T. (2017). The sibsize revolution and social disparities in children's family contexts in the United States, 1940-2012. Demography 54(3): 813-834. doi:10.1007/s13524-017-0568-0.

Featherman, D.L., Jones, F.L., and Hauser, R.M. (1975). Assumptions of social mobility research in the US. The case of occupational status. Social Science Research 4(4): 329-360. doi:10.1016/0049-089X(75)90002-2. 
Ferrari, G. and Dalla-Zuanna, G. (2010). Siblings and human capital. A comparison between Italy and France. Demographic Research S10(21): 587-614. doi:10.4054/DemRes.2010.23.21.

Field, J., Johnson, A.M., Wadsworth, J., and Wellings, K. (1995). National Survey of Sexual Attitudes and Lifestyles 1990. Colchester: UK Data Service. doi:10.5255/ UKDA-SN-3434-1.

Freedman, R., Campbell, A.A., and Whelpton, P.K. (2009). Growth of American families 1955. Ann Arbor: Inter-University Consortium for Political and Social Research. doi:10.3886/ICPSR20000.v2.

Fu, Y.-c. (2015). Taiwan Social Change Survey (Round 6, Year 5). Religion. Taipei: Academia Sinica, Survey Research Data Archive. doi:10.6141/TW-SRDAR090053-1.

Fu, Y.-c. (2017). Taiwan Social Change Survey (Round 7, Year 1). Work Orientation. Taipei: Academia Sinica, Survey Research Data Archive. doi:10.6141/TWSRDA-R090055-1.

Ganzeboom, H.B.G. and Ultee, W. (1993). Family Survey Dutch Population 19921993. The Hague: DANS. doi:10.17026/dans-xhu-j4yz.

Gibbs, B.G., Workman, J., and Downey, D.B. (2016). The (conditional) resource dilution model: State- and community-level modifications. Demography 53(3): 723-748. doi:10.1007/s13524-016-0471-0.

Grätz, M., Barclay, K., Wiborg, Ø.N., Lyngstad, T.H., Karhula, A., Erola, J., Präg, P., Laidley, T., and Conley, D. (2019). Universal family background effects on education across and within societies. Stockholm Research Reports in Demography 2019(13). doi:10.17045/sthlmuni.7999148.v1.

Hajnal, J. (1965). European marriage patterns in perspective. In: Glass, D.V. and Eversley, D.E.C. (eds.). Population in history: Essays in historical demography. London: Edward Arnold: 101-143.

Inaba, A. (2015). National Family Research of Japan 2008. Ann Arbor: InterUniversity Consortium for Political and Social Research. doi:10.3886/ICPSR 36127.v2.

INSEE (1985). Training and Vocational Skills Survey (FQP) 1985. Paris: Archives de Données Issues de la Statistique Publique - Centre Maurice Halbwachs. bdq.quetelet.progedo.fr. 
INSEE (1993). Training and Vocational Skills Survey (FQP) 1993. Paris: Archives de Données Issues de la Statistique Publique - Centre Maurice Halbwachs. bdq.quetelet.progedo.fr.

INSEE (2003). Training and Vocational Skills Survey (FQP) 2003. Paris: Archives de Données Issues de la Statistique Publique - Centre Maurice Halbwachs. bdq.quetelet.progedo.fr.

ISSP Research Group (1988). International Social Survey Program 1986. Social Networks and Support Systems. Cologne: Gesis. doi:10.4232/1.1620.

Jalovaara, M., Neyer, G., Andersson, G., Dahlberg, J., Dommermuth, L., Fallesen, P., and Lappegård, T. (2019). Education, gender, and cohort fertility in the Nordic countries. European Journal of Population 35(3): 563-586. doi:10.1007/s10680018-9492-2.

Johnson, A.M., LSHTM Centre for Sexual and Reproductive Health Research, NatCen Social Research, and Mercer, C.H. (2015). National Survey of Sexual Attitudes and Lifestyles 2010-2012. Colchester: UK Data Service. doi:10.5255/UKDASN-7799-1.

Kalmijn, M., Leeuw, S.G. de, Hornstra, M., Ivanova, K., Gaalen, R. van, and Houdt, K. van (2019). Family complexity into adulthood: The central role of mothers in shaping intergenerational ties. American Sociological Review 84(5): 876-904. doi:10.1177/0003122419871959.

Kim, S.-W. (2014). Korean General Social Survey 2004. Ann Arbor: Inter-University Consortium for Political and Social Research. doi:10.3886/ICPSR34660.v2.

Kim, S.-W., Chang, Y.-H., Iwai, N., and Li, L. (2014). East Asian Social Survey (EASS) Cross-National Survey Data Sets. Families in East Asia, 2006. Ann Arbor: InterUniversity Consortium for Political and Social Research. doi:10.3886/ ICPSR34606.v3.

Köppen, K. and Trappe, H. (2019). The gendered division of labor and its perceived fairness. Implications for childbearing in Germany. Demographic Research 40(48): 1413-1440. doi:10.4054/DemRes.2019.40.48.

Lam, D. and Marteleto, L. (2008). Stages of the demographic transition from a child's perspective. Family size, cohort size, and children's resources. Population and Development Review 34(2): 225-252. doi:10.1111/j.1728-4457.2008.00218.x.

Lipset, S.M. and Bendix, R. (1959). Social mobility in industrial society. Berkeley: University of California Press. 
Logan, J.R. and Bian, Y. (2000). China Housing Survey, 1993. Ann Arbor: InterUniversity Consortium for Political and Social Research. doi:10.3886/ICPSR02 571.v2.

Mare, R.D. (2011). A multigenerational view of inequality. Demography 48(1): 1-23. doi:10.1007/s13524-011-0014-7.

Mare, R.D. and Maralani, V. (2006). The intergenerational effects of changes in women's educational attainments. American Sociological Review 71(4): 542564. doi:10.1177/000312240607100402.

McHale, S.M., Updegraff, K.A., and Whiteman, S.D. (2012). Sibling relationships and influences in childhood and adolescence. Journal of Marriage and Family 74(5): 913-930. doi:10.1111/j.1741-3737.2012.01011.x.

Monden, C., Choi, S., Taiji, R., and Chen, M. (2018). International Sibsize and Educational Attainment Database (ISEAD). Cambridge: Harvard Dataverse. doi:10.7910/DVN/F9PKG5.

National Centre for Social Research, Johnson, A.M., Fenton, K.A., Copas, A.J., Mercer, C.H., McCadden, A., Carder, C., Ridgway, G., Wellings, K., Macdowall, W., and Nanchahal, K. (2005). National Survey of Sexual Attitudes and Lifestyles 2000-2001. Colchester: UK Data Service. doi:10.5255/UKDA-SN-5223-1.

Nisén, J., Martikainen, P., Myrskylä, M., and Silventoinen, K. (2018). Education, other socioeconomic characteristics across the life course, and fertility among Finnish men. European Journal of Population 34(3): 337-366. doi:10.1007/s10680-0179430-8.

Notestein, F.W. (1936). Class differences in fertility. Annals of the American Academy of Political and Social Science 188(1): 26-36. doi:10.1177/00027162361880 0104.

Office of Population Censuses and Surveys (1985). National Heights and Weights Survey 1980. Colchester: UK Data Service. doi:10.5255/UKDA-SN-2046-1.

Oxford Social Mobility Group, Halsey, A.H., Goldthorpe, J.H., Payne, C., and Heath, A. (1978). Social Mobility Inquiry 1972. Colchester: UK Data Service. doi:10.5255/UKDA-SN-1097-1.

Park, B.Y. (2011). Education and social mobility 2008-2011. Seoul: Korean Educational Development Institute. eng.kedi.re.kr. 
Park, H. (2008). Public policy and the effect of sibship size on educational achievement: A comparative study of 20 countries. Social Science Research 37(3): 874-887. doi:10.1016/j.ssresearch.2008.03.002.

Präg, P. and Subramanian, S.V. (2017). Educational inequalities in self-rated health across US States and European countries. International Journal of Public Health 62(6): 709-716. doi:10.1007/s00038-017-0981-6.

Präg, P., Choi, S., and Monden, C. (2020). Replication materials to the sibsize revolution in international context: Declining social disparities in the number of siblings in 26 countries. Open Science Framework. doi:10.17605/OSF.IO/W Y3EG.

Putnam, R.D. (2015). Our kids: The American dream in crisis. New York: Simon \& Schuster.

Schupp, J., Goebel, J., Kroh, M., Schröder, C., Bartels, C., Erhardt, K., Fedorets, A., Giesselmann, M., Grabka, M., Krause, P., Kühne, S., Richter, D., Siegers, R., Schmelzer, P., Schmitt, C., Schnitzlein, D., and Wenzig, K. (2017). German Socio-Economic Panel 1984-2015. Berlin: German Institute for Economic Research. doi:10.5684/soep.v32.

Skirbekk, V. (2008). Fertility trends by social status. Demographic Research 18(5): 145-180. doi:10.4054/DemRes.2008.18.5.

Słomczynski, K.M., Bialecki, I., Domanski, H., Janicka, K., Mach, B.W., Sawinski, Z., Sikorska, J., and Zaborowski, W. (2008). Social structure in Poland 1988. Cologne: Gesis. doi:10.4232/1.4670.

Smith, T.W., Hout, M., and Marsden, P.V. (2016). General Social Survey 1972-2014. Ann Arbor: Inter-University Consortium for Political and Social Research. doi:10.3886/ICPSR36319.v2.

Sobotka, T. (2017). Childlessness in Europe. Reconstructing long-term trends among women born in 1900-1972. In: Kreyenfeld, M. and Konietzka, D. (eds.). Childlessness in Europe: Patterns, causes, and contexts. New York: Springer: 17-53. doi:10.1007/978-3-319-44667-7_2.

Song, X. and Campbell, C.D. (2017). Genealogical microdata and their significance for social science. Annual Review of Sociology 43: 75-99. doi:10.1146/annurev-soc073014-112157.

SSM Survey Management Committee (2011a). Social Stratification and Social Mobility Survey 1995. Tokyo: Social Science Japan Data Archive. ssjda.iss.u-tokyo.ac.jp. 
SSM Survey Management Committee (2011b). Social Stratification and Social Mobility Survey 2005. Tokyo: Social Science Japan Data Archive. ssjda.iss.utokyo.ac.jp.

Statistics Canada (1995). Canadian General Social Survey 1995. Ottawa: Statistics Canada. statcan.gc.ca.

Statistics Canada (2000). Canadian General Social Survey 2000. Ottawa: Statistics Canada. statcan.gc.ca.

Steelman, L.C., Powell, B., Werum, R., and Carter, S. (2002). Reconsidering the effects of sibling configuration: Recent advances and challenges. Annual Review of Sociology 28: 243-269. doi:10.1146/annurev.soc.28.111301.093304.

Swafford, M., Kozyreva, P., Kosolapov, M., and Nizamova, A. (2006). General Social Survey of the Russian Federation and Central Asia October-December 1992. Ann Arbor: Inter-University Consortium for Political and Social Research. doi:10.3886/ICPSR06499.v1.

Szelényi, I. and Treiman, D.J. (2017). Social Stratification in Eastern Europe After 1989. General Population Survey. Cambridge: Harvard Dataverse. doi:1902.1/ M653V1.

Tanioka, I., Iwai, N., and Maeda, Y. (2016). Japanese General Social Survey 2012. Ann Arbor: Inter-University Consortium for Political and Social Research. doi:10.3886/ICPSR36577.v1.

Tanioka, I., Iwai, N., Nitta, M., and Sato, H. (2007a). Japanese General Social Survey 2000. Ann Arbor: Inter-University Consortium for Political and Social Research. doi:10.3886/ICPSR03593.v2.

Tanioka, I., Iwai, N., Nitta, M., and Sato, H. (2007b). Japanese General Social Survey 2001. Ann Arbor: Inter-University Consortium for Political and Social Research (ICPSR). doi:10.3886/ICPSR04213.v2.

Tanioka, I., Iwai, N., Nitta, M., and Sato, H. (2007c). Japanese General Social Survey 2002. Ann Arbor: Inter-University Consortium for Political and Social Research. doi:10.3886/ICPSR04214.v2.

Tanioka, I., Iwai, N., Nitta, M., and Yasuda, T. (2010). Japanese General Social Survey 2006. Ann Arbor: Inter-University Consortium for Political and Social Research. doi:10.3886/ICPSR25181.v1. 
Tanioka, I., Iwai, N., Nitta, M., and Yasuda, T. (2015). Japanese General Social Survey 2008. Ann Arbor: Inter-University Consortium for Political and Social Research (ICPSR). doi:10.3886/ICPSR30661.v3.

Tanioka, I., Maeda, Y., and Iwai, N. (2015). Japanese General Social Survey 2010. Ann Arbor: Inter-University Consortium for Political and Social Research. doi:10.3886/ICPSR34623.v3.

Tanioka, I., Nitta, M., Iwai, N., and Yasuda, T. (2007). Japanese General Social Survey 2005. Ann Arbor: Inter-University Consortium for Political and Social Research (ICPSR). doi:10.3886/ICPSR04703.v1.

Tsuya, N.O., Choe, M.K., Bumpass, L.L., and Rindfuss, R.R. (2009). National Survey on Family and Economic Conditions. Ann Arbor: Inter-University Consortium for Political and Social Research. doi:10.3886/ICPSR21120.v1.

UNESCO (2006). ISCED 1997. International Standard Classification of Education. Montreal: UNESCO Institute for Statistics.

University of Essex, Institute for Social and Economic Research, and NatCen Social Research (2016). Understanding Society. Waves 1-6, 2009-2015. 8th ed. Colchester: UK Data Service. doi:10.5255/UKDA-SN-6614-9.

Van Bavel, J. (2010). Subreplacement fertility in the West before the Baby Boom: Past and current perspectives. Population Studies 64(1): 1-18. doi:10.1080/00324720 903362806 .

Vikat, A., Spéder, Z., Beets, G., Billari, F., Buehler, C., Desesquelles, A., Fokkema, T., Hoem, J.M., MacDonald, A., Neyer, G., Pailhé, A., Pinnelli, A., and Solaz, A. (2007). Generations and Gender Survey (GGS). Towards a better understanding of relationships and processes in the life course. Demographic Research 17(14): 389-440. doi:10.4054/DemRes.2007.17.14.

Whang, I.C., Seok, H., Cha, J.-C., Hong, D., Kim, H.J., Sohn, T.W., Chung, C.-K., and Kim, S.J. (2004). Survey on the Inequality and Justice of Korean Society 1990. Seoul: Korea Social Science Data Archive. A1-1990-0001-Eng.

Whiteman, S.D., McHale, S.M., and Soli, A. (2011). Theoretical perspectives on sibling relationships. Journal of Family Theory and Review 3(2): 124-139. doi:10.1111/ j.1756-2589.2011.00087.x.

Workman, J. (2016). Sibling additions, resource dilution, and cognitive development during early childhood. Journal of Marriage and Family 79(2): 462-474. doi:10.1111/jomf.12350. 
Wright, E.O. (1990). Comparative project on class structure and class consciousness: Core and country-specific files. Ann Arbor: Inter-University Consortium for Political and Social Research. doi:10.3886/ICPSR09323.v1.

Xie, Y., Zhang, X., Tu, P., and Ren, Q. (2016). China Family Panel Studies (CFPS). Ann Arbor: Inter-University Consortium for Political and Social Research. doi:10.3886/ICPSR36524.v1.

Zajonc, R.B. and Markus, G.B. (1975). Birth order and intellectual development. Psychological Review 82(1): 74-88. doi:10.1037/h0076229. 
Präg, Choi \& Monden: The sibsize revolution in international context 